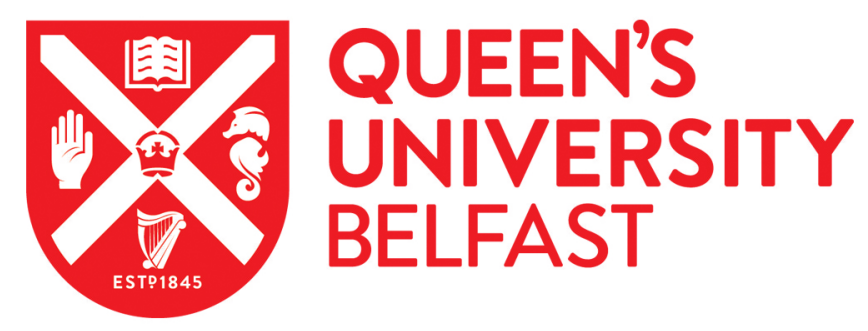

\title{
Exploiting Direct Links in Multiuser Multirelay SWIPT Cooperative Networks with Opportunistic Scheduling
}

Do, N. T., da Costa, D. B., Duong, Q., Bao, V. N. Q., \& An, B. (2017). Exploiting Direct Links in Multiuser Multirelay SWIPT Cooperative Networks with Opportunistic Scheduling. IEEE Transactions on Wireless Communications. https://doi.org/10.1109/TWC.2017.2710307

Published in:

IEEE Transactions on Wireless Communications

Document Version:

Peer reviewed version

Queen's University Belfast - Research Portal:

Link to publication record in Queen's University Belfast Research Portal

Publisher rights

Copyright 2017 IEEE

This work is made available online in accordance with the publisher's policies. Please refer to any applicable terms of use of the publisher.

\section{General rights}

Copyright for the publications made accessible via the Queen's University Belfast Research Portal is retained by the author(s) and / or other copyright owners and it is a condition of accessing these publications that users recognise and abide by the legal requirements associated with these rights.

Take down policy

The Research Portal is Queen's institutional repository that provides access to Queen's research output. Every effort has been made to ensure that content in the Research Portal does not infringe any person's rights, or applicable UK laws. If you discover content in the Research Portal that you believe breaches copyright or violates any law, please contact openaccess@qub.ac.uk. 


\title{
Exploiting Direct Links in Multiuser Multirelay SWIPT Cooperative Networks with Opportunistic Scheduling
}

\author{
Nhu Tri Do, Student Member, IEEE, Daniel Benevides da Costa, Senior Member, IEEE, \\ Trung Q. Duong, Senior Member, IEEE, Vo Nguyen Quoc Bao, Senior Member, IEEE, \\ and Beongku An, Member, IEEE.
}

\begin{abstract}
In this paper, we analyze the downlink outage performance of opportunistic scheduling in dual-hop cooperative networks consisting of one source, multiple radio-frequency (RF) energy harvesting relays, and multiple destinations. To this end, two low-complexity, suboptimal, yet efficient, relay-destination selection schemes are proposed, namely direct links plus opportunistic channel state information (CSI)-based selection (DOS) scheme and direct links plus partial CSI-based selection (DPS) scheme. Considering three relaying strategies, i.e., decode-andforward (DF), variable-gain amplify-and-forward (VG-AF), and fixed-gain amplify-and-forward (FG-AG), the performance analysis in terms of outage probability (OP) is carried out for each selection scheme. For the DF and VG-AF strategies, exact analytical expressions and tight closed-form approximate expressions for the OP are derived. For the FG-AF strategy, an exact closed-form expression for the OP is provided. Additionally, we propose a gradient-based search method to find the optimal values of the power-splitting ratio that minimizes the attained OPs. The developed analysis is corroborated through MonteCarlo simulation. Comparisons with the optimal joint selection scheme are performed and it is shown that the proposed schemes significantly reduce the amount of channel estimations while achieving comparable outage performance. In addition, regardless of relaying strategy used, numerical results show that the DOS scheme achieves full diversity gain, i.e., $M+K$, and the DPS scheme achieves the diversity gain of $M+1$, where $M$
\end{abstract}

This work was presented in part at the IEEE Int. Conf. on Recent Advances in Signal Process. Telecommunications Computing (SigTelCom), Da Nang, Vietnam, Jan. 2017.

The work of N. T. Do and B. An was supported by the Basic Science Research Program through the National Research Foundation of Korea (NRF) funded by the Ministry of Education (Grant No. 2016R1D1A1B03934898) and by the Leading Human Resource Training Program of Regional Neo Industry through the National Research Foundation of Korea (NRF) funded by the Ministry of Science, ICT and Future Planning (Grant No. 2016H1D5A1910577). The work of T. Q. Duong was supported in part by the U.K. Royal Academy of Engineering Research Fellowship under Grant RF1415 $\backslash 14 \backslash 22$ and by the U.K. Engineering and Physical Sciences Research Council (EPSRC) under Grant EP/P019374/1. The work of V. N. Q. Bao is funded by Vietnam National Foundation for Science and Technology Development (NAFOSTED) under grant number 102.04-2014.32

N. T. Do is with the Department of Electronics and Computer Engineering, Hongik University, Sejong 30016, Korea (email: dotrinhu@gmail.com).

D. B. da Costa is with the Department of Computer Engineering, Federal University of Ceará, Sobral, CE, Brazil (email: danielbcosta@ieee.org).

T. Q. Duong is with the Department of Electronics, Electrical Engineering and Computer Science, Queen's University Belfast, Belfast BT7 1NN, U.K. (e-mail: trung.q.duong@qub.ac.uk).

V. N. Q. Bao is with the Department of Telecommunications, Posts and Telecommunications Institute of Technology, HCM City, Vietnam (e-mail: baovnq@ptithcm.edu.vn)

B. An is with the Department of Computer and Information Communications Engineering, Hongik University, Sejong 30016, Korea (email: beongku@hongik.ac.kr). and $K$ are the numbers of destinations and relays, respectively.

Index Terms-Cooperative networks, opportunistic scheduling, outage performance, power-splitting, relay-destination selection, RF energy harvesting, simultaneous wireless information and power transfer (SWIPT).

\section{INTRODUCTION}

Radio-frequency (RF) energy transfer and harvesting techniques have been recognized as sustainable solutions for selfpowering in the next-generation wireless networks [2]. Although wireless power transfer, where energy can be harvested from electromagnetic radiation, has been a long-standing research interest, its applications to wireless communications are still limited. Recently, simultaneous wireless information and power transfer (SWIPT) techniques [3] have attracted attention in the literature. Since RF signals are able to carry both energy and information, the underlying idea behind SWIPT techniques is that users can harvest energy and decode information from their received observations simultaneously. Early ideas of SWIPT were proposed in [4], [5], where the receiver can harvest energy and decode information from the same received signal at the same time. However, these theoretical operations were infeasible due to the limitations of the electric circuits [6]. Some practical SWIPT receiver architectures were proposed in [7], namely time-switching (TS) receiver and power-splitting (PS) receiver architectures, which have been widely adopted in the literature.

Cooperative relaying techniques have been realized as a potential mean to improve coverage and capacity of wireless networks, and have been adopted in industry standards, e.g., the IEEE 802.16j standard for relay-based wireless access networks [8]. Additionally, cooperative relaying techniques can be employed in wide range of wireless networks such as cognitive radio networks [9], [10], space time network codingbased networks [11].

Considering downlink/uplink scenarios of wireless networks where a source communicates with multiple destinations, existing works in the literature have shown that the performance of such networks will be better if the source chooses to communicate with the destination having the best channel condition [12]. In the literature, downlink scenarios of multiuser multirelay cooperative networks have been considered as an example of point-to-multipoint dual-hop systems [13], 
i.e., a single source communicates with multiple destinations with the help of multiple relays. In such systems, opportunistic scheduling, i.e., the source communicates with only one scheduled destination with an assistant of only one selected relay, has been recognized as an attractive scheduling method to improve system performance [13], [14] since the timevarying nature of wireless channels is exploited. However, when the numbers of relays and destinations are large, channel feedbacks, i.e., the amount of CSI estimations, required by the source to select the optimal joint relay-destination becomes significantly large.

While cooperative transmission has the ability to increase the reliability of wireless communications as well as the coverage of wireless networks, the raised question is how to deal with the energy issue at the relays which are often energy-constrained nodes. SWIPT is an appropriate answer since relays can scavenge energy from the source's signal and use this harvested energy to power their relaying operation.

Considering amplify-and-forward (AF) relaying strategy, the authors in [15] studied the outage performance of the proposed TS-based relaying and PS-based relaying protocols for a simple relaying network composed of one source, one RF energy harvesting relay, and one destination. Considering a similar single relay network but adopting a decode-andforward (DF) relaying strategy, maximum transmission rates of the TS-based and PS-based relaying protocols were analyzed in [16] for the cases with and without direct links. In [17], the authors studied SWIPT in multiple-input multiple-output orthogonal frequency-division multiplexing (MIMO-OFDM) relaying system. Adopting non-regenerative relaying protocol, two optimization problems to maximize the end-to-end achievable data rate were addressed when TS receiver and RS receiver, respectively, were employed.

When multiple relays are available, the performance of cooperative transmissions can be improved by selecting only the best relay among available relays that assist the sourcedestination transmission based either on the partial relay selection protocol [18] or on the opportunistic relay selection protocol [19]-[21]. Considering charging/discharging behavior of the battery of relay nodes which is modeled as a twostate Markov chain, several relay selection schemes, i.e., random selection, distance-based selection, and distributed beamforming schemes were rigorously investigated in [22]. Also, considering a source-destination pair communication assisted by multiple RF energy harvesting relays using AF strategy, the author in [23] proposed a relay selection scheme that takes into account the battery status in addition to channel state information (CSI) for making selection criterion.

In addition, multiuser SWIPT cooperative networks which consist of multiple sources and/or multiple destinations that communicate with each other via multiple relays have been studied. In [24], relaying strategies to distribute the harvested energy among multiple source-destination pairs and their impact on the system performance were investigated. In [25], the authors considered a RF energy harvesting relay using the max-min criterion, which is similar to the conventional opportunistic relay selection protocol, to schedule only $m$ out of $M$ source-destination pairs. Also considering multiple source-destination pairs that communicating through their dedicated PS-based energy harvesting relays, the authors in [26] adopted game theory to develop a distributed PS framework for SWIPT in relay interference channels. In particular, using game-theoretical approach, the authors proposed algorithms to find the optimal value of the power-splitting ratio that maximize the achievable data rate when AF, DF, and hybrid AF/DF relaying strategies were employed. Recently, in [27], the authors investigated the applications of PS-based SWIPT to large-scale multicell networks, where a base station of each cell communicates with its cell-edge user via an AF relay. Specifically, the authors developed a resource allocation scheme that jointly optimizes base station transmit power, PS factors, and relay transmit power. However, the above mentioned related works, e.g., [15], [17], [22]-[27], did not take the effect of the direct $\operatorname{link}(s)$ between source(s) and destination(s) into account.

In [28], the authors studied RF energy harvesting in a wireless-powered cooperative communication network, which consists of a hybrid access-point (AP), a hybrid relay, and an information source. Assuming the direct link between the AP and the source is available, the authors developed two cooperative protocols, namely energy cooperation protocol and dual cooperation protocol. With the same assumption of the availability of the direct link, the authors in [29] proposed a harvest-then-cooperate protocol for a cooperative networks consisting of a hybrid AP, a source, and a relay. Specifically, in downlink transmission, the source and the relay harvest energy from the AP using TS-based architecture, while in uplink transmission, the source transmits data to the AP with the help of the relay under delay-limited transmission mode. Considering a cooperative network with one sourcedestination pair and multiple SWIPT relays where the direct link between the source and the destination exists, the authors in [30] proposed three relay selection schemes, and then two distributed game theoretic algorithms were developed for the case of multiple sources.

Different from the aforementioned works on (multiuser) multirelay cooperative networks, e.g., [22]-[25], [30], which adopted the assumption of independent and identically distributed (i.i.d.) fading channels, there are few existing works considering independent and not-necessarily identically distributed (i.n.i.d.) fading channels, specially in cooperative relaying networks with SWIPT. In [31], the authors studied ergodic and outage capacities of a three-node relaying network (source-relay-destination) in which the relay harvests energy from source and co-channel interference (CCI) signals, where the CCI signals are assumed to be i.n.i.d. channels. In [32], the authors investigated the best relay selection scheme in cooperative cognitive relaying networks, where before data transmission the destination uses spectrum sensing to decide whether primary user is active or not.

In this paper, we consider a more general SWIPT cooperative relaying network, i.e., multiuser multirelay cooperative networks under i.n.i.d. fading channels where the direct links between the source and the destinations are available. More specifically, the purpose of this paper is to propose new opportunistic scheduling schemes to alleviate considerably 
the number of CSI estimations and comparisons of potential links compared to the optimal joint relay-destination selection (OJRDS) scheme. It is noteworthy that the performance analysis of the OJRDS scheme in multiuser multirelay cooperative networks with SWIPT has not been carried out in the literature since such performance analysis is intractable. To this end, two suboptimal relay-destination selection schemes are proposed, namely direct links plus opportunistic CSI-based selection (DOS) scheme, and direct links plus partial CSI-based selection (DPS) scheme. Specifically, both schemes exploit the direct links between the source and destinations to select the strongest destination scheduled in each transmission block. Only one relay is then selected to help the selected destination. In particular, in the DOS scheme, a relay that maximizes endto-end signal-to-noise ratios (SNRs) of dual-hop links will be selected. On the other hand, in the DPS scheme, a relay that maximizes SNRs of first-hop links ${ }^{1}$ will be selected.

It is noteworthy to point out that the motivation of proposing the DOS and DPS schemes is that, in addition to achieve comparable outage performance with the OJRDS scheme, their operation not only require less channel feedbacks, i.e., the amount of channel estimations, but also lower complexity, i.e., the number of comparisons of potential links to find out appropriate relay-destination pair, as will be discussed in details in Section VI.

In addition, in [25], the authors recently concluded that the use of the max-min criterion, i.e., choosing opportunistically a relay that maximizes the end-to-end SNR of dual-hop links, results in loss of diversity gains in SWIPT cooperative networks. In particular, the diversity gain achieved by the maxmin criterion is $(K+1) / 2$ for the scenario with one sourcedestination pair and $K$ RF energy harvesting relays. In this paper, we show that when both direct links and opportunistic relay selection protocol are utilized in the proposed DOS scheme, the diversity gain of SWIPT cooperative networks is not jeopardized, i.e., the DOS scheme provides full diversity gain.

Moreover, in [30], it was shown that the diversity gain achieved by a relay selection based on second-order statistics of source-relay links is always 2 for the scenario with one source-destination pair and $K \mathrm{RF}$ energy harvesting relays. The second-order statistics was determined by the distance between source and relay, and the source-destination direct link was assumed to be available. In this paper, considering multiple destinations instead of only one as in [30], and by utilizing the SNR statistics of direct links together with sourcerelay links, diversity gain can be improved as the number of destinations increases, i.e., the diversity gain of the DPS scheme is $M+1$.

With the above motivations, the main contributions of the paper are summarized as follows:

- We propose two suboptimal, yet efficient, relaydestination selection schemes, i.e., DOS and DPS

\footnotetext{
${ }^{1}$ The reason of considering the SNRs of first-hop links is that, in SWIPT, the first-hop links play a more important role than the second-hop links since the first-hop links impact not only on the received SNR at a relay but also on the transmit power that the relay uses to forward the source's information to the destination [25].
}

schemes, for downlink scenarios of multiuser multirelay SWIPT cooperative networks with opportunistic scheduling. The DOS scheme is able to reduce drastically the number of CSI estimations compared to that required by the OJRDS scheme while still achieves full diversity gain. On the other hand, the DPS scheme reduces even more channel estimations compared to that needed for the DOS scheme while achieves efficient diversity gain compared to that of previous existing schemes.

- We carry out comprehensive outage performance analyses of the proposed schemes by considering three well-known relaying strategies, i.e., DF, VG-AF, and FG-AF. To provide insights into the impact of system parameters on the performance of the proposed schemes, exact analytical expressions and tight closed-form approximate expressions for the OP of the DF and VG-AF strategies, and exact closed-form expression for the OP of the FG-AF strategy, are provided for each scheme. It is noteworthy that the attained closed-form approximate expressions of OPs can predict accurately the exact ones for each considered scenario in low as well as high SNR regime.

- The developed analysis is corroborated through MonteCarlo simulations and some representative performance comparisons are presented. On one hand, we show that the DOS scheme is diversity-optimal in multiuser multirelay SWIPT cooperative networks, and the achievable diversity gain is $M+K$. On the other hand, the diversity gain of the DPS scheme only depends on $M$ and is independent of $K$. In particular, the diversity gain achieved by the DPS scheme is $M+1$.

- We conduct an optimal analysis of the power-splitting ratio $\rho$ (used in the power-splitting receiver architecture). To this end, we propose the gradient-based search method to find the optimal values of $\rho$, denoted by $\rho^{*}$, that minimize the attained OPs. With the proposed algorithm, $\rho^{*}$ can be found offline (before the data transmission).

The rest of the paper is arranged as follows. Section II introduces the system model and describes in detail the relay-destination selection processes of the DPS and DOS schemes. Section III presents the analytical results in terms of the OP for the considered scenarios, and the proofs of the analytical expressions are provided in the appendixes. Section IV presents the optimal analysis of the power-splitting ratio. Section VI presents some illustrative numerical results, based on which insightful discussions are provided. Monte Carlo simulations are shown to corroborate the proposed analysis. Finally, Section VII concludes the paper.

\section{System Model And Selection Schemes DESCRIPTIONS}

\section{A. System Model}

Consider a downlink scenario of a dual-hop cooperative network composed of one source node S (e.g., base station or access point), a set of $K$ RF energy harvesting (EH) relays $\mathrm{R}_{\text {set }}=\left\{\mathrm{R}_{k} \mid k=1, \ldots, K\right\}$, and a set of $M$ available destinations $\mathrm{D}_{\text {set }}=\left\{\mathrm{D}_{m} \mid m=1, \ldots, M\right\}$ as sketched in Fig. 1. We assume that all nodes are equipped with one antenna 
and operate in half-duplex mode and over the same frequency band. A time-division multiple-access scheme is used for orthogonal access, and we assume perfect synchronization in the network [7]. All wireless links are assumed to undergo independent but not necessarily identically distributed (i.n.i.d.) Rayleigh block flat fading.

The operation in each transmission block is divided into two processes, namely relay-destination selection process and communication process.

- The relay-destination selection process is performed before the communication process and will be based on the respective channel gains. This selection process is conducted through the signaling and CSI estimation/calculation system $^{2}$ and is based on a selection criterion of the DOS scheme or the DPS scheme, which will be described in the next subsection.

- After the relay-destination pair is selected ${ }^{3}$, the communication process is carried out in two phases. In the first phase, the source broadcasts the selected destination's message. The destination receives the source's signal without using SWIPT technique, while the relay performs SWIPT technique for energy harvesting and information processing from its received observation as depicted in Fig. 2. In the second phase, the relay uses the energy that harvested during the first phase to forward the received signal to the destination using either DF, VG-AF, or FGAF relaying strategies. The destination then combines the direct signal and the relaying signal using selection combining (SC) technique.

In the sequel of the paper, let $h_{\mathrm{XY}}$ and $\left|h_{\mathrm{XY}}\right|^{2}$ denote the channel coefficient of $\mathrm{X} \rightarrow \mathrm{Y}$ channel and the corresponding channel gain, respectively, let $n_{\mathrm{Y}}$ denote the additive white Gaussian noise (AWGN) at $\mathrm{Y}$ where $\mathrm{X} \in\{\mathrm{S}\} \cup \mathrm{R}_{\text {set }}$ and $Y \in R_{\text {set }} \cup D_{\text {set }}$. Without loss of generality, herein, we assume that all terminals have the same AWGN mean power $N_{0}$ and all wireless channels in the network exhibit Rayleigh fading. Thus, the channel gain $\left|h_{\mathrm{XY}}\right|^{2}$ is an exponential random variable with probability density function (PDF), $f_{\left|h_{\mathrm{XY}}\right|^{2}}(z)=\lambda_{\mathrm{XY}} e^{-\lambda_{\mathrm{XY}} z}$, where $\lambda_{\mathrm{XY}}$ denotes the rate parameter, i.e., $\lambda_{\mathrm{XY}}=d_{\mathrm{XY}}^{\epsilon}$, where $d_{\mathrm{XY}}$ represents the distance between two nodes (in meter) and $\epsilon$ stands for pathloss exponent. Thus, the average channel gain can be presented as $\mathrm{E}\left[\left|h_{\mathrm{XY}}\right|^{2}\right]=\frac{\mathcal{L}}{\left(d_{\mathrm{XY}} / d_{0}\right)^{\epsilon}}$, where $d_{0}$ denotes the reference distance and $\mathcal{L}$ is the average signal power attenuation at $d_{0}$.

\section{B. Relay-Destination Selection Process}

Considering RF energy harvesting, we assume that all relays have the same energy conversion efficiency $\eta$ [29], [33], which is known at the source. In addition, we consider a static power-splitting receiver architecture with power-splitting ratio $\rho$ as in [7], [33], [34]. Since the variation of $\rho$ affects the

\footnotetext{
${ }^{2}$ As will be better explained next, the relays use their own energies to acquire/estimate the respective CSIs so that the energy harvested from the source's signal is employed only during the communication process.

${ }^{3}$ The unselected destinations will seek a chance to communicate in the next transmission block while the unselected relays can be back to their own duties, e.g., keep silence, harvest energy from ambient RF signals, or transmit their own data.
}

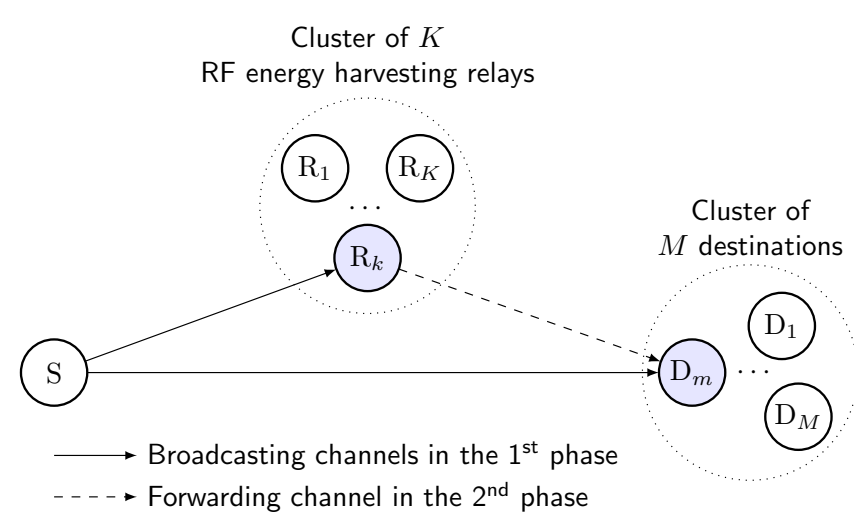

Fig. 1. Schematic illustration of multiuser multirelay SWIPT cooperative networks.

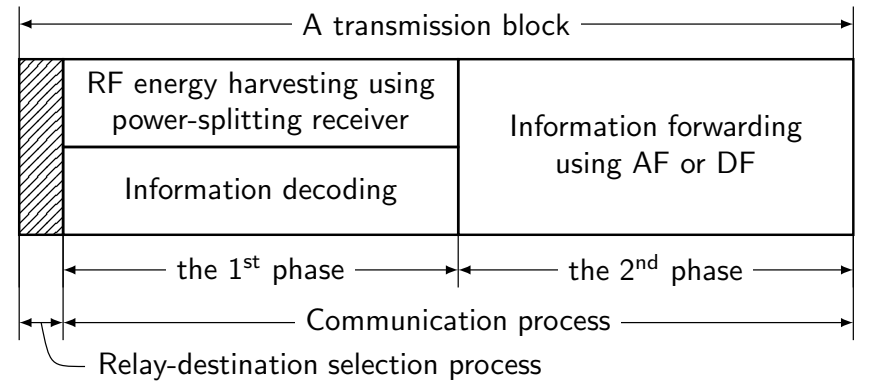

Fig. 2. Schematic illustration of relaying operation at the selected relay with SWIPT technique.

system performance, as will be shown in the numerical plots, this parameter is assumed to be controlled by the source. A similar assumption was made in [29], in which an access point controls the fraction of time for harvesting energy in timeswitching receiver architecture.

1) Selection Criterion of The DOS Scheme: Let $\bar{\gamma} \triangleq P_{\mathrm{S}} / N_{0}$ denote the transmit SNR of the source. The DOS scheme first selects the best destination as follows

$$
\mathrm{D}_{\mathrm{b}}=\arg \max _{1 \leq m \leq M}\left|h_{\mathrm{SD}_{m}}\right|^{2},
$$

and then selects the best relay as follows

$$
\mathrm{R}_{\mathrm{b}}=\arg \max _{1 \leq k \leq K} \min \left\{(1-\rho) \bar{\gamma}\left|h_{\mathrm{SR}_{k}}\right|^{2}, \eta \rho \bar{\gamma}\left|h_{\mathrm{SR}_{k}}\right|^{2}\left|h_{\mathrm{R}_{k} \mathrm{D}_{\mathrm{b}}}\right|^{2}\right\},
$$

for the case of using DF relaying strategy, and

$$
\begin{aligned}
& \mathrm{R}_{\mathrm{b}}= \\
& \arg \max _{1 \leq k \leq K}\left\{\frac{\eta \rho(1-\rho) \bar{\gamma}^{2}\left|h_{\mathrm{SR}_{k}}\right|^{4}\left|h_{\mathrm{R}_{k} \mathrm{D}_{\mathrm{b}}}\right|^{2}}{(1-\rho) \bar{\gamma}\left|h_{\mathrm{SR}_{k}}\right|^{2}+\eta \rho \bar{\gamma}\left|h_{\mathrm{SR}_{k}}\right|^{2}\left|h_{\mathrm{R}_{k} \mathrm{D}_{\mathrm{b}}}\right|^{2}+1}\right\},
\end{aligned}
$$

for the case of using VG-AF relaying strategy, and

$$
\mathrm{R}_{\mathrm{b}}=\arg \max _{k}\left\{\frac{\eta \rho(1-\rho) \bar{\gamma}^{2}\left|h_{\mathrm{SR}_{k}}\right|^{4}\left|h_{\mathrm{R}_{k} \mathrm{D}_{\mathrm{b}}}\right|^{2}}{\kappa_{k}\left|h_{\mathrm{SR}_{k}}\right|^{2}+\eta \rho \bar{\gamma}\left|h_{\mathrm{SR}_{k}}\right|^{2}\left|h_{\mathrm{R}_{k} \mathrm{D}_{\mathrm{b}}}\right|^{2}}\right\},
$$

for the case of using FG-AF relaying strategy, where $\kappa_{k}$ is a function of the Euclidean distance between the source and the relay $k$, which will be explained in details in Theorem 3 . 
2) Selection Criterion of The DPS Scheme: The DPS scheme first selects the best destination as follows

$$
\mathrm{D}_{\mathrm{b}}=\arg \max _{1 \leq m \leq M}\left|h_{\mathrm{SD}_{m}}\right|^{2},
$$

and then simply uses the same selection criterion for all the cases of the DF, VG-AF, and FG-AF strategies to select the best relay as follows

$$
\mathrm{R}_{\mathrm{b}}=\arg \max _{1 \leq k \leq K}\left|h_{\mathrm{SR}_{k}}\right|^{2} .
$$

As can be observed, the DPS scheme can reduce the number of required CSI estimations compared to the DOS schemes. In addition, the DPS scheme performs a simple comparator, which turns out a lower number of comparisons of potential links, to select the best relay. Hence, our work provides a flexible choice for system design as follows: if the source has a powerful computational capability, the DOS scheme can be used since it achieves higher outage performance than that of the DPS scheme, otherwise, the DPS scheme can be used not only to reduce the implementation complexity but also to provide comparable outage performance.

We assume that the source and the relays use their own energy to transmit pilot signal to estimate channel conditions. For the sake of simplicity, and as an initial work in this field dealing with the proposed system setup, a simple power allocation strategy, i.e., $P_{\mathrm{R}_{k}}=P_{\mathrm{S}}$, can be used in this process. As will be explained in the next sub-section, the harvested energy will be used by a selected relay during data transmission process.

Moreover, a brief discussion on the fairness of the proposed selection scheme is provided next. Regarding the fairness of relays, in the proposed schemes, the relay-destination selection process is conducted before the data transmission. Therefore, after this process, the non-selected relays are assumed to be free, they can keep silence, harvest energy for their own purpose, or transmit their own data. On the other hand, if a node is not available to serve as a relay (e.g., it has its own data to send, critical battery level for channel estimation, etc.), it will leave the relay set. Regarding the fairness of destinations, we introduce a so-called occupation limitation, i.e., the maximum consecutive transmission slots that a destination can seize. In particular, if a certain destination continuously has the best channel condition, and if the number of transmission slot it holds reaches the occupation limitation, it has to release the channel, set a back-off time to keep silence. However, performance analysis of such fairness issues needs different analysis framework, such as using Markov model as in [22], and is out of the scope of our paper.

\section{Communication Process}

The communication process is carried out in two phases. Without loss of generality, we assume that each phase, i.e., the first and second phases, has the equal time duration of $T$ as in [15], [29], [35]. In the first phase, $S$ broadcasts the selected destination's message $s$ to both $\mathrm{R}_{\mathrm{b}}$ and $\mathrm{D}_{\mathrm{b}}$, where $\mathbb{E}\left[|s|^{2}\right]=1$, $\mathbb{E}[\cdot]$ represents the expectation operator. The received signal at
$\mathrm{D}_{\mathrm{b}}$ can be expressed as $y_{\mathrm{SD}_{\mathrm{b}}}=\sqrt{P_{\mathrm{S}}} h_{\mathrm{SD}_{\mathrm{b}}} s+n_{\mathrm{D}_{\mathrm{b}}}$. Thus, the received SNR of the $\mathrm{S} \rightarrow \mathrm{D}_{\mathrm{b}}$ channel, $\gamma_{\mathrm{SD}_{\mathrm{b}}}$, can be given by

$$
\gamma_{\mathrm{SD}_{\mathrm{b}}}=\bar{\gamma}\left|h_{\mathrm{SD}_{\mathrm{b}}}\right|^{2} .
$$

In this paper, the source and destination nodes are assumed unconstrained powered nodes, while the selected relay node powers itself by using SWIPT technique when it relays the source information to the selected destination. As mentioned earlier, the static power-splitting receiver architecture with power-splitting ratio $\rho, 0<\rho<1$, is utilized [7, Sec. III], [33], [34]. Moreover, in this paper, we consider the pessimistic case of PS receiver where the power splitting receiver only utilizes the signal power, but not the antenna noise power, as done in [24], [35], [36]. In particular, the received power from the source's signal is divided into two streams with the splitting ratio $\rho$ and $(1-\rho)$ respectively, with $0<\rho<1$. The first stream, i.e., $\rho$ portion of the received power, is used for energy harvesting while the remaining stream, i.e., $(1-\rho)$ portion of the received power, is used for information processing. Thus, the harvested energy at the selected relay, $E_{\mathrm{R}_{\mathrm{b}}}$, can be given by $E_{\mathrm{R}_{\mathrm{b}}}=\eta \rho P_{\mathrm{S}}\left|h_{\mathrm{SR}_{\mathrm{b}}}\right|^{2} T$ [16], [35], where $0<\eta<1$ denotes the energy conversion efficiency; and the remaining part of the received signal for information processing at $\mathrm{R}_{\mathrm{b}}$ can be expressed as $y_{\mathrm{SR}_{\mathrm{b}}}=\sqrt{(1-\rho) P_{\mathrm{S}}} h_{\mathrm{SR}_{\mathrm{b}}} s+n_{\mathrm{R}_{\mathrm{b}}}$. Thus, the received SNR of the $\mathrm{S} \rightarrow \mathrm{R}_{\mathrm{b}}$ channel, $\gamma_{\mathrm{SR}_{\mathrm{b}}}$, can be given by

$$
\gamma_{\mathrm{SR}_{\mathrm{b}}}=(1-\rho) \bar{\gamma}\left|h_{\mathrm{SR}_{\mathrm{b}}}\right|^{2} .
$$

In the second phase, $R_{b}$ is supposed to use all the harvested power ${ }^{4}$ during the first phase from the source's signal to forward the source information to $\mathrm{D}_{\mathrm{b}}$ [15], [16], [35]. Thus, transmit power of $\mathrm{R}_{\mathrm{b}}$ can be expressed as $P_{\mathrm{R}_{\mathrm{b}}}=E_{\mathrm{R}_{\mathrm{b}}} / T=$ $\eta \rho P_{\mathrm{S}}\left|h_{\mathrm{SR}_{\mathrm{b}}}\right|^{2}$.

In this phase, three relaying strategies are then considered at $\mathrm{R}_{\mathrm{b}}$ to perform the relaying operation, namely decode-andforward (DF), variable-gain amplify-and-forward (VG-AF), and fixed-gain amplify-and-forward (FG-AF) strategies. An AF relay simply amplifies the received signal and forwards the resulting signal to the destination, whereas a DF relay decodes the source's information, re-encodes and then forwards to the destination. Hence, while the received noise is also amplified and forwarded to the destination when using AF strategy, it can be eliminated when using DF strategy. On the other hand, an amplification factor used in AF strategy can be determined based on the instantaneous or statistical average (mean) of the channel gain of the source-relay link, which is known as VG-AF or FG-AF, respectively [37]. As can be seen, the principle of operation of FG-AF strategy is less complex than that of VG-AF strategy since the former one uses a constant amplification factor. However, VG-AF strategy may be more suitable for EH relaying networks than FG-AF strategy since the harvested energy (which will be used to amplify the source's signal) at the relay is not constant and

\footnotetext{
${ }^{4}$ In this paper, we assume that there is no energy accumulation in $R_{b}$ so that all the collected energy is employed to forward the source information to $\mathrm{D}_{\mathrm{b}}$. Energy accumulation is indeed beyond the scope of our paper, but it arises as an interesting issue for being investigated in future works.
} 
also depends on the instantaneous channel gain of the sourcerelay link. Next, three mentioned relaying strategies will be mathematically described.

1) DF Relaying Strategy: Suppose that $R_{b}$ uses $D F$ relaying strategy to forward the source information to $\mathrm{D}_{\mathrm{b}}$, and provided that the relay can successfully decode the information from the source ${ }^{5}$, the received signals at $\mathrm{D}_{\mathrm{b}}$ are given by $y_{\mathrm{R}_{\mathrm{b}} \mathrm{D}_{\mathrm{b}}}^{\mathrm{DF}}=\sqrt{P_{\mathrm{R}_{\mathrm{b}}}} h_{\mathrm{R}_{\mathrm{b}} \mathrm{D}_{\mathrm{b}}} \hat{s}+n_{\mathrm{D}_{\mathrm{b}}}$, where $\hat{s}$ denotes the reencoded version of $s$. Thus, the received SNR of the $\mathrm{R}_{\mathrm{b}} \rightarrow \mathrm{D}_{\mathrm{b}}$ channel, $\gamma_{\mathrm{R}_{\mathrm{b}} \mathrm{D}_{\mathrm{b}}}$, can be written as

$$
\gamma_{\mathrm{R}_{\mathrm{b}} \mathrm{D}_{\mathrm{b}}}=\eta \rho \bar{\gamma}\left|h_{\mathrm{SR}_{\mathrm{b}}}\right|^{2}\left|h_{\mathrm{R}_{\mathrm{b}} \mathrm{D}_{\mathrm{b}}}\right|^{2} \text {. }
$$

Note that, although $\gamma_{R_{b} D_{b}}$ results from the case of DF strategy, it will be used in the sequel of the analysis and for the VG-AF and FG-AF strategies since it generally represents the received SNR of the second-hop of the dual-hop links.

In dual-hop DF transmission, the failure in one of the two hops leads to the failure of the transmission. Thus, from (8) and (9), the end-to-end SNR of the $\mathrm{S} \rightarrow \underset{\mathrm{R}_{\mathrm{b}} \rightarrow \mathrm{D}_{\mathrm{b}}}{\rightarrow}$ channel achieved by the DF relaying strategy, $\gamma_{S_{2} R_{b} D_{b}}^{e 2 e, D F}$, can be expressed as

$$
\gamma_{\mathrm{SR}_{\mathrm{b}} \mathrm{D}_{\mathrm{b}}}^{\mathrm{e} 2 \mathrm{D}, \mathrm{m}}=\min \left\{\gamma_{\mathrm{SR}_{\mathrm{b}}}, \gamma_{\mathrm{R}_{\mathrm{b}} \mathrm{D}_{\mathrm{b}}}\right\}
$$

2) AF Relaying Strategy: Considering an AF relaying strategy, the best relay first amplifies the received source information signal by an amplification factor $G \in\left\{G_{v}, G_{f}\right\}$ and then forwards it to the best destination. The received signal at $\mathrm{D}_{\mathrm{b}}$ is given by $y_{\mathrm{R}_{\mathrm{b}} \mathrm{D}_{\mathrm{b}}}^{\mathrm{AF}}=G h_{\mathrm{R}_{\mathrm{b}} \mathrm{D}_{\mathrm{b}}} y_{\mathrm{SR}_{\mathrm{b}}}+n_{\mathrm{D}_{\mathrm{b}}}=$ $\sqrt{(1-\rho) P_{\mathrm{S}}} G h_{\mathrm{SR}_{\mathrm{b}}} h_{\mathrm{R}_{\mathrm{b}} \mathrm{D}_{\mathrm{b}}} s+G h_{\mathrm{R}_{\mathrm{b}} \mathrm{D}_{\mathrm{b}}} n_{\mathrm{R}_{\mathrm{b}}}+n_{\mathrm{D}_{\mathrm{b}}}$. Thus, the end-to-end SNR achieved by the AF relaying strategy through the best path can be given by

$$
\begin{aligned}
\gamma_{\mathrm{SR}_{\mathrm{b}} D_{\mathrm{b}}}^{\mathrm{e} 2 \mathrm{e}, \mathrm{AF}} & =\frac{(1-\rho) P_{\mathrm{S}} G^{2}\left|h_{\mathrm{SR}_{\mathrm{b}}}\right|^{2}\left|h_{\mathrm{R}_{\mathrm{b}} \mathrm{D}_{\mathrm{b}}}\right|^{2} \frac{P_{\mathrm{R}_{\mathrm{b}}}}{G^{2} N_{0}^{2}}}{\left(G^{2}\left|h_{\mathrm{R}_{\mathrm{b}} \mathrm{D}_{\mathrm{b}}}\right|^{2} N_{0}+N_{0}\right) \frac{P_{\mathrm{R}_{\mathrm{b}}}}{G^{2} N_{0}^{2}}} \\
& =\frac{\frac{(1-\rho) P_{\mathrm{S}}\left|h_{\mathrm{SR}_{\mathrm{b}}}\right|^{2}}{N_{0}} \frac{P_{\mathrm{R}_{\mathrm{b}}}\left|h_{\mathrm{R}_{\mathrm{b}} \mathrm{D}_{\mathrm{b}}}\right|^{2}}{N_{0}}}{\frac{P_{\mathrm{R}_{\mathrm{b}}}\left|h_{\mathrm{R}_{\mathrm{b}} \mathrm{D}_{\mathrm{b}}}\right|^{2}}{N_{0}}+\frac{P_{\mathrm{R}_{\mathrm{b}}}}{G^{2} N_{0}}} \\
& =\frac{\gamma_{\mathrm{SR}_{\mathrm{b}}} \gamma_{\mathrm{R}_{\mathrm{b}} \mathrm{D}_{\mathrm{b}}}}{\gamma_{\mathrm{R}_{\mathrm{b}} \mathrm{D}_{\mathrm{b}}}+\frac{P_{\mathrm{R}_{\mathrm{b}}}}{G^{2} N_{0}}} .
\end{aligned}
$$

In the VG-AF strategy, the amplification factor $G_{v}$ can be calculated based on the instantaneous CSI of the $\mathrm{S} \rightarrow \mathrm{R}_{\mathrm{b}}$ channel and can be written as $G_{v}=\sqrt{\frac{P_{\mathrm{R}_{\mathrm{b}}}}{P_{\mathrm{S}}\left|h_{\mathrm{SR}_{\mathrm{b}}}\right|^{2}+N_{0}}}$ [37]. Thus, the end-to-end SNR achieved by the VG-AF strategy can be expressed as

$$
\gamma_{\mathrm{SR}_{\mathrm{b}} \mathrm{D}_{\mathrm{b}}}^{\mathrm{e} 2 \mathrm{e}, \mathrm{VG}-\mathrm{AF}}=\frac{\gamma_{\mathrm{SR}_{\mathrm{b}}} \gamma_{\mathrm{R}_{\mathrm{b}} \mathrm{D}_{\mathrm{b}}}}{\gamma_{\mathrm{SR}_{\mathrm{b}}}+\gamma_{\mathrm{R}_{\mathrm{b}} \mathrm{D}_{\mathrm{b}}}+1}
$$

In the FG-AF strategy ${ }^{6}$, the factor $G_{f}$ can be calculated based only on the statistical channel information, i.e., the

\footnotetext{
${ }^{5}$ For a fair comparison with amplify-and-forward (AF) relaying strategy, we assume that the selected relay always correctly decodes its received signal, as considered in previous works [15], [16]. For further readings, the studies that considered whether the relay successfully decodes its receive signal or not can be found in [22], [23], [38]

${ }^{6}$ Note that the amplification factor of the FG-AF strategy uses statistical CSI to determine the transmit power of the selected relay. Consequently, the transmit power of the relay could not be well adjusted according to the channel fluctuations, which leads to a performance degradation when compared to the variable-gain amplify-and-forward (VG-AF) strategy, as shown in Section VI.
}

statistical average (mean) of the channel gains [37]. In the case of FG-AF strategy, the statistical average of the channel gain of the $\mathrm{S} \rightarrow \mathrm{R}_{\mathrm{b}}$ channel is assumed to be obtained at the selected relay so that the amplification factor $G_{f}$ can be written as $G_{f}=\sqrt{\frac{\eta \rho P_{\mathrm{S}} \mathbb{E}\left[\left|h_{\mathrm{SR}_{\mathrm{b}}}\right|^{2}\right]}{P_{\mathrm{S}} \mathbb{E}\left[\left|h_{\mathrm{SR}_{\mathrm{b}}}\right|^{2}\right]+N_{0}}}$. Since $\left|h_{\mathrm{SR}_{\mathrm{b}}}\right|^{2}$ follows exponential distribution with rate parameter $\lambda_{\mathrm{SR}_{\mathrm{b}}}$, where, $\lambda_{\mathrm{SR}_{\mathrm{b}}}=d_{\mathrm{SR}_{\mathrm{b}}}^{\epsilon}$, the statistical average of the channel gain is determined as $\mathbb{E}\left[\left|h_{\mathrm{SR}_{\mathrm{b}}}\right|^{2}\right]=\frac{1}{\lambda_{\mathrm{SR}_{\mathrm{b}}}}$. Thus, the amplification factor for the FGAF strategy can be given by $G_{f}^{2}=\frac{\eta \rho \bar{\gamma}\left(1 / \lambda_{\mathrm{SR}_{\mathrm{b}}}\right)}{\bar{\gamma}\left(1 / \lambda_{\mathrm{SR}_{\mathrm{b}}}\right)+1}$. Hence, the end-to-end SNR achieved by the FG-AF strategy can be given by

$$
\gamma_{\mathrm{SR} \mathrm{b}_{\mathrm{b}}}^{\mathrm{e} 2 \mathrm{e}, \mathrm{FG}-\mathrm{AF}}=\frac{\gamma_{\mathrm{SR}_{\mathrm{b}}} \gamma_{\mathrm{R}_{\mathrm{b}} \mathrm{D}_{\mathrm{b}}}}{\kappa_{\mathrm{b}}\left|h_{\mathrm{SR}_{\mathrm{b}}}\right|^{2}+\gamma_{\mathrm{R}_{\mathrm{b}} \mathrm{D}_{\mathrm{b}}}}
$$

where $\kappa_{\mathrm{b}}=\bar{\gamma}+\lambda_{\mathrm{SR}_{\mathrm{b}}}=\bar{\gamma}+d_{\mathrm{SR}_{\mathrm{b}}}^{\epsilon}$, which is a function of the Euclidean distance between $\mathrm{S}$ and $\mathrm{R}_{\mathrm{b}}$.

Finally, in the selection combining phase, a SC strategy ${ }^{7}$ is conducted at $\mathrm{D}_{\mathrm{b}}$ to select the best path, i.e., $\mathrm{S} \rightarrow \mathrm{D}_{\mathrm{b}}$ or $\mathrm{S} \rightarrow \mathrm{R}_{\mathrm{b}} \rightarrow \mathrm{D}_{\mathrm{b}}$ paths. Thus, the end-to-end SNR of the system can be determined as

$$
\gamma_{\text {system }}^{\mathrm{e} 2 \mathrm{e}, \mathrm{str}}=\max \left\{\gamma_{\mathrm{SD}_{\mathrm{b}}}, \gamma_{\mathrm{SR}_{\mathrm{b}} \mathrm{D}_{\mathrm{b}}}^{\mathrm{e} 2 \mathrm{e}, \mathrm{str}}\right\}
$$

where strategy $\in\{\mathrm{DF}, \mathrm{VG}-\mathrm{AF}, \mathrm{FG}-\mathrm{AF}\}$.

\section{Outage Performance Analysis}

In this section, we evaluate the performance for the proposed relay-destination selection schemes, i.e., DOS and DPS schemes, in terms of the OP. Such a metric can be defined as the probability that the end-to-end SNR of the system falls below a SNR threshold $\gamma_{\text {th }}$, i.e., OP $=\operatorname{Pr}\left(\gamma_{\text {system }}^{\text {e2e,strategy }}<\gamma_{\text {th }}\right)$, where $\gamma_{\mathrm{th}}=2^{2 R_{\mathrm{th}}}-1$, and $R_{\mathrm{th}}$ denotes the target data rate, and $\operatorname{Pr}(A)$ is the probability of an event $A$.

For the sake of notational convenience, in the sequel of the paper, let $\alpha \triangleq(1-\rho) \bar{\gamma}, \beta \triangleq \eta \rho \bar{\gamma}$, and $\mu \triangleq \gamma_{\text {th }} / \alpha$. Thus, the SNRs in (8) and (9) can be rewritten as $\gamma_{\mathrm{SR}_{\mathrm{b}}}=\alpha\left|h_{\mathrm{SR}_{\mathrm{b}}}\right|^{2}$, $\gamma_{\mathrm{R}_{\mathrm{b}} \mathrm{D}_{\mathrm{b}}}=\beta\left|h_{\mathrm{SR}_{\mathrm{b}}}\right|^{2}\left|h_{\mathrm{R}_{\mathrm{b}} \mathrm{D}_{\mathrm{b}}}\right|^{2}$. Additionally, $\Omega, \Theta_{m}$, and $\Xi_{k}$ are defined in (66), (67), and (68), respectively. Let $\widetilde{\Sigma}_{l}=$ $\sum_{l=1}^{K}(-1)^{l+1} \sum_{\substack{q_{1}=1 \\ q_{1}<\cdots<q_{l}}}^{K} \cdots \sum_{\substack{q_{l}=1 \\ K}}^{K}$ and $\widehat{\sum}_{t}=\sum_{t=1}^{l} \lambda_{\mathrm{SR}_{q_{t}}}$.

\section{A. DOS Scheme}

1) DF Relaying Strategy: From (2), (10), and (14), the OP achieved by the DOS scheme assuming DF relaying can be formulated as

$$
\begin{aligned}
& P_{\text {out }}^{\text {DOS DF }} \\
& \quad=\operatorname{Pr}\left(\max \left\{\gamma_{\mathrm{SD}_{\mathrm{b}}}, \max _{1 \leq k \leq K} \min \left\{\gamma_{\mathrm{SR}_{k}}, \gamma_{\mathrm{R}_{k} \mathrm{D}_{\mathrm{b}}}\right\}\right\}<\gamma_{\mathrm{th}}\right) .
\end{aligned}
$$

\footnotetext{
${ }^{7}$ The reason of using SC instead of maximal-ratio combining (MRC) is due to complexity issues and to avoid CSI estimation error effects as well. Thus, it is reasonable for us to assume a perfect CSI estimation in this paper.
} 
Theorem 1: An analytical expression for the OP of the DOS scheme using DF relaying can be given by

$$
\begin{aligned}
& P_{\text {out }}^{\mathrm{DOS}, \mathrm{DF}} \\
& =\Omega \sum_{m=1}^{M} \Theta_{m} \prod_{k=1}^{K}[1-\lambda_{\mathrm{SR}_{k}} \underbrace{\int_{\mu}^{\infty} e^{-y \lambda_{\mathrm{SR}_{k}}-\frac{\gamma_{\mathrm{th} \lambda_{\mathrm{R}} \mathrm{D}_{m}}}{\beta y}} d y}_{\Lambda_{\mathrm{DOS}}}] .
\end{aligned}
$$

Proof: See Appendix A.

To the best of authors' knowledge, the integral $\Lambda_{\mathrm{DOS}}$ in (16) cannot be simplified. The following lemma that relies on the Maclaurin series enables us to derive a tractable form for the result presented in Theorem 1.

Lemma 1: For $\theta, \xi>0$, the integral $\Lambda \triangleq \int_{\mu}^{\infty} e^{-\theta x-\frac{\xi}{x}} d x$ can be given by

$$
\begin{aligned}
\Lambda \approx & \frac{e^{-\mu \theta}}{\theta}-\xi \Gamma(0, \mu \theta)+\sum_{u=2}^{\infty} \frac{(-1)^{u} \xi^{u}}{u !} \\
& \times\left[e^{-\mu \theta} \sum_{v=1}^{u-1} \frac{(v-1) !(-\theta)^{u-v-1}}{(u-1) ! \mu^{v}}-\frac{(-\theta)^{u-1}}{(u-1) !} \operatorname{Ei}(-\mu \theta)\right],
\end{aligned}
$$

where $\Gamma(\cdot, \cdot)$ is the upper incomplete Gamma function [39, Eq. (8.350.2)], $\operatorname{Ei}(\cdot)$ is the exponential integral function [39, Eq. (8.211.1)].

Proof: Using the Maclaurin series ${ }^{8}$ of the term $e^{-\frac{\xi}{x}}=$ $\sum_{u=0}^{\infty} \frac{(-1)^{u} \xi^{u}}{u ! x^{u}}$, then, by making use of the formula [39, Eq. (3.381.3)] and [39, Eq. (3.353.1)], and after some algebraic manipulations, (17) can be obtained. This completes the proof.

Now, applying Lemma 1, a closed-form approximate expression for the OP of the DOS scheme using DF relaying can be derived as

$$
\begin{aligned}
P_{\text {out,approx }}^{\text {DOS,DF }} & \\
= & \Omega \sum_{m=1}^{M} \Theta_{m} \prod_{k=1}^{K}\left[1-\theta_{1}\left[\frac{e^{-\mu \theta_{1}}}{\theta_{1}}-\xi_{1} \Gamma\left(0, \mu \theta_{1}\right)\right.\right. \\
& +\sum_{u=2}^{9} \frac{(-1)^{u} \xi_{1}^{u}}{u !}\left[e^{-\mu \theta_{1}} \sum_{v=1}^{u-1} \frac{(v-1) !\left(-\theta_{1}\right)^{u-v-1}}{(u-1) ! \mu^{v}}\right. \\
& \left.\left.\left.-\frac{\left(-\theta_{1}\right)^{u-1}}{(u-1) !} \operatorname{Ei}\left(-\mu \theta_{1}\right)\right]\right]\right]
\end{aligned}
$$

where $\theta_{1}=\lambda_{\mathrm{SR}_{k}}$, and $\xi_{1}=\frac{\gamma_{\mathrm{th}} \lambda_{\mathrm{R}_{k} \mathrm{D}_{m}}}{\beta}$.

2) VG-AF Relaying Strategy: From (3), (12), and (14), the OP of the DOS scheme assuming VG-AF relaying can be given by

$$
\begin{aligned}
& P_{\mathrm{out}}^{\mathrm{DOS}, \mathrm{VG}-\mathrm{AF}} \\
& =\operatorname{Pr}\left(\max \left\{\gamma_{\mathrm{SD}_{\mathrm{b}}}, \max _{1 \leq k \leq K}\left\{\frac{\gamma_{\mathrm{SR}_{k}} \gamma_{\mathrm{R}_{k} \mathrm{D}_{\mathrm{b}}}}{\gamma_{\mathrm{SR}_{k}}+\gamma_{\mathrm{R}_{k} \mathrm{D}_{\mathrm{b}}}+1}\right\}\right\}<\gamma_{\mathrm{th}}\right) .
\end{aligned}
$$

\footnotetext{
${ }^{8}$ It is noted that when we increase the number of terms in the Maclaurin expansion of $\frac{\xi}{x}$, the corresponding closed-form approximate expression for the OP provides a very accurate result compared to the exact one.
}

Theorem 2: An analytical expression for the OP of the DOS scheme using VG-AF relaying can be derived as

$$
\begin{aligned}
P_{\mathrm{out}}^{\mathrm{DOS}, \mathrm{VG}-\mathrm{AF}}= & \Omega \sum_{m=1}^{M} \Theta_{m} \prod_{k=1}^{K}\left[1-\lambda_{\mathrm{SR}_{k}}\right. \\
& \times \underbrace{\int_{\mu}^{\infty} e^{-y \lambda_{\mathrm{SR}_{k}}-\frac{\left(\alpha \gamma_{\mathrm{th}} y+\gamma_{\mathrm{th}}\right) \lambda_{\mathrm{R}_{k} \mathrm{D} m}}{\left(\alpha y-\gamma_{\mathrm{th}}\right) \beta y}} d y}_{\Psi_{\mathrm{DOS}}} .
\end{aligned}
$$

Proof: See Appendix B.

Note that the integral $\Psi_{\mathrm{DOS}}$ in (20) does not have a closedform expression. Next, approximation technique will be used to derive a more tractable form for (31). Here, for two real positive numbers, $a$ and $b$, we have $\frac{a b}{a+b+1} \approx \frac{a b}{a+b}$ when $a$ and $b$ are sufficient large. Thus, the end-to-end SNR $\gamma_{\mathrm{SR}_{\mathrm{B}} \mathrm{V} \mathrm{DG}-\mathrm{AF}}^{\mathrm{e}}$ in (12) can be approximated by

$$
\begin{aligned}
\gamma_{\mathrm{SR}_{\mathrm{b}} \mathrm{D}_{\mathrm{b}}}^{\mathrm{e} 2 \mathrm{e}-\mathrm{AF}} & \approx \frac{\gamma_{\mathrm{SR}_{\mathrm{b}}} \gamma_{\mathrm{R}_{\mathrm{b}} \mathrm{D}_{\mathrm{b}}}}{\gamma_{\mathrm{SR}_{\mathrm{b}}}+\gamma_{\mathrm{R}_{\mathrm{b}} \mathrm{D}_{\mathrm{b}}}} \\
& =\max _{1 \leq k \leq K} \frac{\gamma_{\mathrm{SR}_{k}} \gamma_{\mathrm{R}_{k} \mathrm{D}_{\mathrm{b}}}}{\gamma_{\mathrm{SR}_{k}}+\gamma_{\mathrm{R}_{k} \mathrm{D}_{\mathrm{b}}}}
\end{aligned}
$$

Plugging (21) into (19) and then carrying out the similar steps as done in Appendix B, the integral $\Psi_{\mathrm{DOS}}$ in (20) can be approximated by

$$
\Psi_{\mathrm{DOS}} \approx \int_{\mu}^{\infty} e^{-y \lambda_{\mathrm{SR}_{k}}-\frac{\alpha \gamma_{\mathrm{th}} \lambda_{\mathrm{R}} \mathrm{D} m}{\left(\alpha y-\gamma_{\mathrm{th}}\right) \beta}} d y
$$

Lemma 2: For $c \neq 0$, the integral $\Psi=a \int_{d / c}^{\infty} e^{-a x-\frac{b}{c x-d}} d x$ can be given by

$$
\Psi=e^{-a d / c} \sqrt{\frac{4 a b}{c}} K_{1}\left(\sqrt{\frac{4 a b}{c}}\right),
$$

where $K_{n}(\cdot)$ is the $n$-order modified Bessel function of the second kind [39, Eq. 8.432.6].

Proof: Let $z=c x-d$, and after some manipulations, $\Psi$ can be re-expressed as $\Psi=\frac{a}{c} e^{-\frac{a d}{c}} \int_{0}^{\infty} e^{-\frac{a z}{c}-\frac{b}{z}} d z$. By making the use of [39, Eq. (3.324.1)], (23) can be obtained, which completes the proof of Lemma 2.

Now, plugging (22) into (20), and then applying Lemma 2 for the case of $a=\lambda_{\mathrm{SR}_{k}}, b=\alpha \gamma_{\mathrm{th}} \lambda_{\mathrm{R}_{k} \mathrm{D}_{m}}, c=\alpha \beta$, and $d=\gamma_{\mathrm{th}} \beta$, a closed-form approximate expression for the OP of the DOS scheme using VG-AF relaying can be attained as

$$
P_{\text {out,approx }}^{\mathrm{DOS}, \mathrm{AG}-\mathrm{AF}}=\Omega \sum_{m=1}^{M} \Theta_{m} \prod_{k=1}^{K}\left[1-e^{-\mu \lambda_{\mathrm{SR}_{k}}} \xi_{2} K_{1}\left(\xi_{2}\right)\right],
$$

where $\xi_{2}=\sqrt{4 \gamma_{\mathrm{th}} \lambda_{\mathrm{SR}_{k}} \lambda_{\mathrm{R}_{k} \mathrm{D}_{m}} / \beta}$.

3) FG-AF Relaying Strategy: From (4), (13), and (14), the $\mathrm{OP}$ of the DOS scheme assuming FG-AF relaying can be given by

$$
\begin{aligned}
& P_{\text {out, exact }}^{\mathrm{DOS}, \mathrm{AF}} \\
& =\operatorname{Pr}\left(\max \left\{\gamma_{\mathrm{SD}_{\mathrm{b}}}, \max _{1 \leq k \leq K}\left\{\frac{\gamma_{\mathrm{SR}_{k}} \gamma_{\mathrm{R}_{k} \mathrm{D}_{\mathrm{b}}}}{\kappa_{k}\left|h_{\mathrm{SR}_{k}}\right|^{2}+\gamma_{\mathrm{R}_{k} \mathrm{D}_{\mathrm{b}}}}\right\}\right\}<\gamma_{\mathrm{th}}\right),
\end{aligned}
$$


Theorem 3: An exact closed-form expression for the OP of the DOS scheme using FG-AF relaying can be given by

$$
P_{\text {out, exact }}^{\mathrm{DOS}, \mathrm{AF}}=\Omega \sum_{m=1}^{M} \Theta_{m} \prod_{k=1}^{K}\left[1-e^{-\mu \lambda_{\mathrm{SR}_{k}}} \xi_{3} K_{1}\left(\xi_{3}\right)\right],
$$

where $\xi_{3}=\sqrt{\left(4 \kappa_{k} \gamma_{\mathrm{th}} \lambda_{\mathrm{SR}_{k}} \lambda_{\mathrm{R}_{k} \mathrm{D}_{m}}\right) /(\alpha \beta)}$ and $\kappa_{k}=\bar{\gamma}+$ $\lambda_{\mathrm{SR}_{k}}$.

Proof: See Appendix C.

Note that for the case of using the FG-AF strategy in both DOS and DPS schemes, closed-form expressions for the OPs can be obtained, while in the cases of the DF and VG-AF strategies, only closed-form approximate expressions for the OPs are available. It is also noteworthy that, as can be showed in Section VI, the approximate results are almost corroborated with the analytical exact results in low as well as high SNR regimes with any value of $\rho \in(0,1)$.

\section{B. DPS Scheme}

1) DF Relaying Strategy: From (10) and (14), the OP of the DPS scheme assuming DF relaying can be formulated as

$$
P_{\text {out }}^{\text {DPS,DF }}=\operatorname{Pr}\left(\max \left\{\gamma_{\mathrm{SD}_{\mathrm{b}}}, \min \left\{\gamma_{\mathrm{SR}_{\mathrm{b}}}, \gamma_{\mathrm{R}_{\mathrm{b}} \mathrm{D}_{\mathrm{b}}}\right\}\right\}<\gamma_{\text {th }}\right) \text {. }
$$

Theorem 4: The analytical expression for the OP of the DPS scheme using DF relaying can be given by

$$
\begin{aligned}
P_{\text {out }}^{\mathrm{DPS}, \mathrm{DF}}= & \Omega\left[1-\sum_{m=1}^{M} \Theta_{m} \sum_{k=1}^{K} \Xi_{k} \widetilde{\sum_{l}} \widehat{\sum_{t}}\right. \\
& \times \underbrace{\int_{\gamma_{\mathrm{th}} / \alpha}^{\infty} e^{-x{\widehat{\sum_{t}}}_{t}-\frac{\gamma_{\mathrm{th}} \lambda_{\mathrm{R}_{k} \mathrm{D}}}{\beta x}} d x}_{\Lambda_{\mathrm{DPS}}} .
\end{aligned}
$$

Proof: See Appendix D.

As can be seen, the integral $\Lambda_{\text {DPS }}$ in (28) has a similar form to that of (16), which does not have a closed-form expression. Thus, the similar approximation method presented in subsection III-A1 will be used to obtain a more tractable form for (28). Specifically, by applying Lemma 1, a closedform approximation for the OP of the DPS scheme using DF strategy can be derived as

$$
\begin{aligned}
P_{\text {out,approx }}^{\text {DPS,DF }} & \Omega\left[1-\sum_{m=1}^{M} \Theta_{m} \sum_{k=1}^{K} \Xi_{k} \widetilde{\sum_{l}} \theta_{4}\left[\frac{e^{-\mu \theta_{4}}}{\theta_{4}}-\xi_{4} \Gamma\left(0, \mu \theta_{4}\right)\right.\right. \\
= & +\sum_{u=2}^{9} \frac{(-1)^{u} \xi_{4}^{u}}{u !}\left[e^{-\mu \theta_{4}} \sum_{v=1}^{u-1} \frac{(v-1) !\left(-\theta_{4}\right)^{u-v-1}}{(u-1) ! \mu^{v}}\right. \\
& \left.\left.-\frac{\left(-\theta_{4}\right)^{u-1}}{(u-1) !} \operatorname{Ei}\left(-\mu \theta_{4}\right)\right]\right]
\end{aligned}
$$

where $\theta_{4}=\widehat{\sum}_{t}$, and $\xi_{4}=\frac{\gamma_{\mathrm{th}} \lambda_{\mathrm{R}_{k} \mathrm{D}_{m}}}{\beta}$.

2) VG-AF Relaying Strategy: From (12) and (14), the OP of the DPS scheme assuming VG-AF relaying can be formulated as

$$
\begin{aligned}
& P_{\mathrm{out}}^{\mathrm{DPS}, \mathrm{VG}-\mathrm{AF}} \\
& \quad=\operatorname{Pr}\left(\max \left\{\gamma_{\mathrm{SD}_{\mathrm{b}}}, \frac{\gamma_{\mathrm{SR}_{\mathrm{b}}} \gamma_{\mathrm{R}_{\mathrm{b}} \mathrm{D}_{\mathrm{b}}}}{\gamma_{\mathrm{SR}_{\mathrm{b}}}+\gamma_{\mathrm{R}_{\mathrm{b}} \mathrm{D}_{\mathrm{b}}}+1}\right\}<\gamma_{\mathrm{th}}\right) .
\end{aligned}
$$

Theorem 5: The analytical expression for the OP of the DPS scheme using VG-AF relaying can be given by

$$
\begin{aligned}
P_{\mathrm{out}}^{\mathrm{DPS}, \mathrm{VG}-\mathrm{AF}}= & \Omega \sum_{m=1}^{M} \Theta_{m} \sum_{k=1}^{K} \Xi_{k}\left[1-\widetilde{\sum_{l}} \widehat{\sum_{t}}\right. \\
& \times \underbrace{\int_{\mu}^{\infty} e^{-x \widehat{\sum}_{t}-\frac{\left(\alpha \gamma_{\mathrm{th}} x+\gamma_{\mathrm{th}}\right) \lambda_{\mathrm{R}_{k} \mathrm{D}_{m}}}{\alpha \beta x^{2}-\beta \gamma_{\mathrm{th}} x}} d x}_{\Psi_{\mathrm{DPS}}} .
\end{aligned}
$$

Proof: See Appendix E.

As can be seen, the integral $\Psi_{\text {DPS }}$ in (31) has a similar form of $\Psi_{\text {DOS }}$ presented in (20), which does not have a closedform expression. Thus, the similar approximation method as done in subsection III-A2 will be adopted to approximate (31). Specifically, plugging the approximated SNR in (21) into (19), and following a similar rationale as done in subsection III-A2, the integral $\Psi_{\text {DPS }}$ in (31) can be approximated by

$$
\Psi_{\mathrm{DPS}} \approx \int_{\gamma_{\mathrm{th}} / \alpha}^{\infty} e^{-x \widehat{\sum}_{t}-\frac{\alpha \gamma_{\mathrm{th}} \lambda_{\mathrm{R}} \mathrm{D}_{m}}{\beta\left(\alpha x-\gamma_{\mathrm{hh}}\right)}}
$$

By plugging (32) into (31) and then applying Lemma 2 for the case of $a=\widehat{\sum}_{t}, b=\alpha \gamma_{\mathrm{th}} \lambda_{\mathrm{R}_{k} \mathrm{D}_{m}}, c=\alpha \beta$, and $d=\gamma_{\mathrm{th}} \beta$, a closed-form approximate expression for the OP of the DPS scheme using VG-AF relaying can be derived as

$$
\begin{aligned}
& P_{\text {out,approx }}^{\text {DPS,VG-AF }} \\
& \quad=\Omega \sum_{m=1}^{M} \Theta_{m} \sum_{k=1}^{K} \Xi_{k}\left[1-\widetilde{\sum_{l}} e^{-\frac{\gamma_{\text {th }} \widehat{\Sigma}_{t}}{\alpha}} \xi_{5} K_{1}\left(\xi_{5}\right)\right],
\end{aligned}
$$

where $\xi_{5}=\sqrt{\left(4 \gamma_{\mathrm{th}} \lambda_{\mathrm{R}_{k} \mathrm{D}_{m}} \widehat{\sum}_{t}\right) / \beta}$.

3) FG-AF Relaying Strategy: From (13) and (14), the OP of the DPS scheme assuming FG-AF relaying can be formulated as

$$
\begin{aligned}
& P_{\text {out,exact }}^{\mathrm{DPS}, \mathrm{FG}} \\
& \quad=\operatorname{Pr}\left(\max \left\{\gamma_{\mathrm{SD}_{\mathrm{b}}}, \frac{\gamma_{\mathrm{SR}_{\mathrm{b}}} \gamma_{\mathrm{R}_{\mathrm{b}} \mathrm{D}_{\mathrm{b}}}}{\kappa_{\mathrm{b}}\left|h_{\mathrm{SR}_{\mathrm{b}}}\right|^{2}+\gamma_{\mathrm{R}_{\mathrm{b}} \mathrm{D}_{\mathrm{b}}}}\right\}<\gamma_{\mathrm{th}}\right) .
\end{aligned}
$$

The following theorem provides an exact closed-form expression for the OP of the FG-AF relaying strategy.

Theorem 6: An exact closed-form expression for the OP of the DPS scheme using FG-AF relaying can be written as

$$
\begin{aligned}
& P_{\text {out,exact }}^{\text {DPS,FG-AF }} \\
& \quad=\Omega \sum_{m=1}^{M} \Theta_{m} \sum_{k=1}^{K} \Xi_{k}\left[1-\widetilde{\sum_{l}} e^{-\frac{\gamma_{\mathrm{th}} \widehat{\Sigma}_{t}}{\alpha}} \xi_{6} K_{1}\left(\xi_{6}\right)\right], \\
& \text { where } \xi_{6}=\sqrt{\frac{4 \kappa_{k} \gamma_{\mathrm{th}} \lambda_{\mathrm{R}_{k} \mathrm{D}_{m} \widehat{\Sigma}_{t}}}{\alpha \beta}} \text { and } \kappa_{k}=\bar{\gamma}+\lambda_{\mathrm{SR}_{k}} .
\end{aligned}
$$

Proof: See Appendix F.

\section{Optimal Analysis of The Power-Splitting RATIO $\rho$}

In this Section, we carry out the optimal analysis of the power-splitting ratio $\rho$. More specifically, adopting the gradient algorithm [40], we propose an gradient-based algorithm to find the optimal value of $\rho$, denoted by $\rho^{*}$, that results in the minimum value of the OPs as follows. 
We first formulate the equivalent minimizing functions $P_{\text {out,minimizing }}^{\text {sch,str }}(\rho)$ for each considered case, where sch $\epsilon$ $\{\mathrm{DOS}, \mathrm{DPS}\}$ and $\mathrm{str} \in\{\mathrm{DF}, \mathrm{VG}-\mathrm{AF}, \mathrm{FG}-\mathrm{AF}\}$. To alleviate notation, let $P_{\text {out }}(\rho)$ represent $P_{\text {out,minimizing }}^{\text {sch,str }}(\rho)$ in the sequel of the paper.

In our paper, the optimization problem concerns to find the optimal value of $\rho \in(0,1)$ that results in a minimum value of an outage probability $P_{\text {out }}(\rho)$. Our algorithm, which is based on the gradient method, can be explained as follows:

- For a given starting value $\rho_{k}$, we first determine a descent direction so that $P_{\text {out }}\left(\rho_{k}\right)>P_{\text {out }}\left(\rho_{k+1}\right)$, where $k$ indicates the iteration index. Considering the gradient method, an obvious choice for the search direction is the negative gradient [40], i.e., $\Delta \rho_{k}=-\nabla P_{\text {out }}(\rho)$, where $\Delta \rho_{k}$ is call step or search direction.

- We next update the value of $\rho_{k}$, i.e., $\rho_{k+1}=\rho_{k}+s_{z} \Delta \rho_{k}$, where $s_{z}>0$ indicates the step size. As can be observed, $\rho_{k}<\rho_{k+1}$ while $P_{\text {out }}\left(\rho_{k}\right)>P_{\text {out }}\left(\rho_{k+1}\right)$, except when $\rho_{k}$ is optimal.

- As we know that when $\rho_{k}$ converges to its optimal value, $\Delta \rho_{k}$ becomes smaller (please note that $\nabla P_{\text {out }}\left(\rho^{*}\right)=0$ ). Therefore, the iteration process of calculating $\Delta \rho_{k}$ and updating $\rho_{k}$ will be stopped when $\Delta \rho_{k}$ is less than a predefined stopping threshold $s_{t}$.

Next, the gradient-based algorithm for finding optimal value of the power-splitting ratio, $\rho *$, can be expressed as on the top of the next page. Please note that with the proposed algorithm, the optimization process can be done offline based on the system parameters acquired through CSI estimation process (and before the data transmission process).

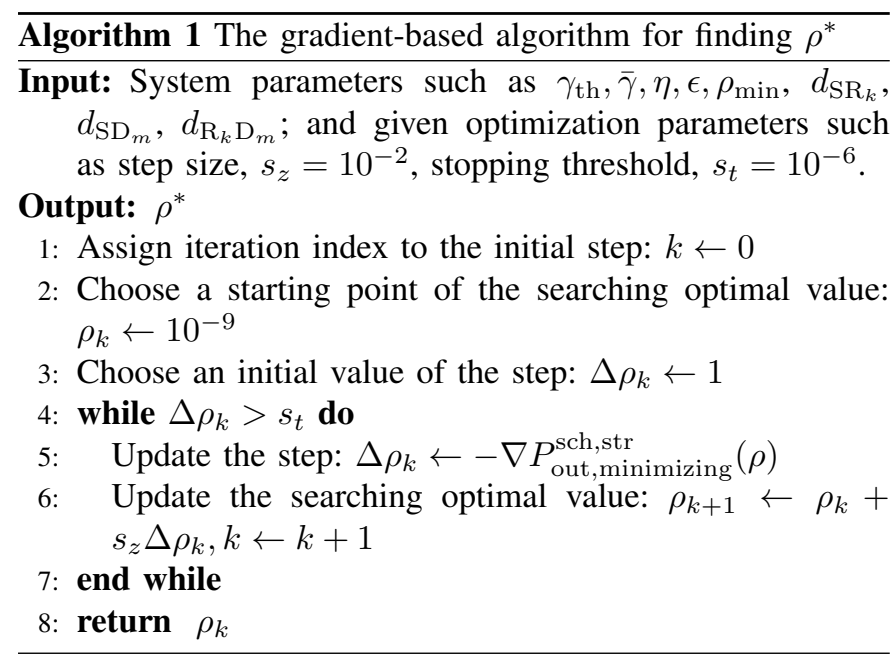

\section{A. DOS DF}

For the case of the DOS scheme using DF relaying, minimizing $P_{\text {out,approx }}^{\mathrm{DOS}, \mathrm{DF}}$ with respect to $\rho$ is equivalent to minimizing $P_{\text {out,minimizing }}^{\mathrm{DOS}, \mathrm{DF}}(\rho)$, which can be expressed as

$$
P_{\text {out,minimizing }}^{\mathrm{DOS}, \mathrm{DF}}(\rho)=\sum_{m=1}^{M} \prod_{k=1}^{K} J_{k, m}^{\mathrm{DOS}, \mathrm{DF}}(\rho) \text {, }
$$

where $J_{k, m}^{\mathrm{DOS}, \mathrm{DF}}(\rho)$ can be written as

$$
\begin{aligned}
& J_{k, m}^{\mathrm{DOS}, \mathrm{DF}}(\rho) \\
& =1-e^{-\frac{a_{1}}{1-\rho}}-\frac{b_{1} \theta_{1}}{\rho} \operatorname{Ei}\left(-\frac{a_{1}}{1-\rho}\right) \\
& \quad+\sum_{u=2}^{9} \sum_{v=1}^{u-1} \frac{(-1)^{u}(v-1) ! b_{1}^{u}\left(-n u_{1}\right)^{u-1}}{(u-1) ! u !\left(\gamma_{\mathrm{th}} / \bar{\gamma}\right)^{v}} \frac{(1-\rho)^{v} e^{-\frac{a_{1}}{1-\rho}}}{\rho^{u}} \\
& \quad \times-\sum_{u=2}^{9} \frac{(-1)^{u} b_{1}^{u}\left(-\theta_{1}\right)^{u}}{(u-1) ! u ! \rho^{u}} \operatorname{Ei}\left(\frac{a_{1}}{1-\rho}\right)
\end{aligned}
$$

where $a_{1}=\frac{\gamma_{\mathrm{th}} \lambda_{\mathrm{SR}_{k}}}{\bar{\gamma}}, b_{1}=\frac{\gamma_{\mathrm{th}} \lambda_{\mathrm{R}_{k} \mathrm{D}_{m}}}{\bar{\gamma} \eta}$, and $\theta_{1}=\lambda_{\mathrm{SR}_{k}}$.

The gradient of $P_{\text {out,minimizing }}^{\mathrm{DOS}, \mathrm{DF}}(\rho), \nabla P_{\text {out,minimizing }}^{\mathrm{DOS}, \mathrm{DF}}(\rho)$, can be obtained as

$$
\begin{aligned}
& \nabla P_{\text {out,minimizing }}^{\mathrm{DOS}, \mathrm{DF}}(\rho) \\
& \quad=\sum_{m=1}^{M} \sum_{k=1}^{K} \dot{J}_{k, m}^{\mathrm{DOS}, \mathrm{DF}}(\rho) \prod_{l=1, l \neq k}^{K} J_{l, m}^{\mathrm{DOS}, \mathrm{DF}}(\rho),
\end{aligned}
$$

where the over dot denotes the first-order derivative of $J_{k, m}^{\mathrm{DOS}, \mathrm{DF}}(\rho)$ with respect to $\rho$, and $\dot{J}_{k, m}^{\mathrm{DOS}, \mathrm{DF}}(\rho)$ can be expressed as

$$
\begin{aligned}
& \dot{J}_{k, m}^{\mathrm{DOS}, \mathrm{DF}}(\rho) \\
&=\frac{a_{1}}{(1-\rho)^{2}} e^{-\frac{a_{1}}{1-\rho}}-b_{1} \theta_{1}\left[\frac{e^{-\frac{a_{1}}{1-\rho}}}{(1-\rho) \rho}-\frac{1}{\rho^{2}} \operatorname{Ei}\left(-\frac{a_{1}}{1-\rho}\right)\right] \\
&-\sum_{u=2}^{9} \sum_{v=1}^{u-1} \frac{(-1)^{u}(v-1) ! b_{1}^{u}\left(-n u_{1}\right)^{u-1}}{(u-1) ! u !\left(\gamma_{\mathrm{th}} / \bar{\gamma}\right)^{v}} e^{-\frac{a_{1}}{1-\rho}} \\
& \times\left[\frac{u(1-\rho)^{v}}{\rho^{u+1}}+\frac{a_{1}(1-\rho)^{v-2}+v(1-\rho)^{v-1}}{\rho^{u}}\right] \\
&-\sum_{u=2}^{9} \frac{(-1)^{u} b_{1}^{u}\left(-\theta_{1}\right)^{u}}{(u-1) ! u !}\left[\frac{1}{(1-\rho) \rho^{u}} e^{-\frac{a_{1}}{1-\rho}}\right. \\
&\left.-\frac{u}{\rho^{u+1}} \operatorname{Ei}\left(-\frac{a_{1}}{1-\rho}\right)\right] .
\end{aligned}
$$

\section{B. DOS VG-AF}

For the case of the DOS scheme using VG-AF relaying, minimizing $P_{\text {out,approx }}^{\mathrm{DOS}, \mathrm{AG}}$ with respect to $\rho$ is equivalent to minimizing $P_{\text {out,minimizing }}^{\mathrm{DO}, \mathrm{VG}-\mathrm{AF}}(\rho)$, which can be expressed as

$$
P_{\text {out,minimizing }}^{\mathrm{DOS}, \mathrm{VG}-\mathrm{AF}}(\rho)=\sum_{m=1}^{M} \prod_{k=1}^{K} \underbrace{\left[1-e^{-\frac{a_{2}}{1-\rho}} \sqrt{\frac{b_{2}}{\rho}} K_{1}\left(\sqrt{\frac{b_{2}}{\rho}}\right)\right]}_{J_{k, m}^{\mathrm{DOS}, \mathrm{VG}-\mathrm{AF}}(\rho)},
$$

where $a_{2}=\frac{\gamma_{\mathrm{th}} \lambda_{\mathrm{SR}_{k}}}{\bar{\gamma}}$ and $b_{2}=\frac{4 \gamma_{\mathrm{th}} \lambda_{\mathrm{SR}_{k}} \lambda_{\mathrm{R}_{k} \mathrm{D}_{m}}}{\eta \bar{\gamma}}$.

The gradient of $P_{\text {out,minimizing }}^{\mathrm{DOS}, \mathrm{VG}-\mathrm{AF}}(\rho), \nabla P_{\text {out,minimizing }}^{\mathrm{DOS}, \mathrm{VG}-\mathrm{AF}}(\rho)$, can be obtained as

$$
\begin{aligned}
& \nabla P_{\text {out,minimizing }}^{\mathrm{DOS}, \mathrm{VG}-\mathrm{AF}}(\rho) \\
& \quad=\sum_{m=1}^{M} \sum_{k=1}^{K} \dot{J}_{k, m}^{\mathrm{DOS}, \mathrm{VG}-\mathrm{AF}}(\rho) \prod_{l=1, l \neq k}^{K} J_{l, m}^{\mathrm{DOS}, \mathrm{VG}-\mathrm{AF}}(\rho),
\end{aligned}
$$


where

$$
\begin{aligned}
\dot{J}_{k, m}^{\mathrm{DOS}, \mathrm{VG}-\mathrm{AF}}(\rho) & =\frac{e^{-\frac{a_{2}}{1-\rho}} b_{2}}{2 \rho^{2}}\left(\sqrt{\frac{b_{2}}{\rho}}\right)^{-1} K_{1}\left(\sqrt{\frac{b_{2}}{\rho}}\right) \\
& -\frac{e^{-\frac{a_{2}}{1-\rho}} b_{2}}{4 \rho^{2}}\left[K_{0}\left(\sqrt{\frac{b_{2}}{\rho}}\right)+K_{2}\left(\sqrt{\frac{b_{2}}{\rho}}\right)\right] \\
& +\frac{e^{-\frac{a_{2}}{1-\rho}} a_{2}}{(1-\rho)^{2}} \sqrt{\frac{b_{2}}{\rho}} K_{1}\left(\sqrt{\frac{b_{2}}{\rho}}\right) .
\end{aligned}
$$

\section{DOS FG-AF}

For the case of the DOS scheme using FG-AF relaying, minimizing $P_{\text {out.exact }}^{\mathrm{DOS}, \mathrm{AF}}$ with respect to $\rho$ is equivalent to minimizing $P_{\text {out,minimizing }}^{\mathrm{DO}, \mathrm{FG}}(\rho)$, which can be expressed as

$$
\begin{aligned}
& P_{\text {out,minimizing }}^{\mathrm{DOS}, \mathrm{FG}-\mathrm{AF}}(\rho) \\
& =\sum_{m=1}^{M} \prod_{k=1}^{K} \underbrace{\left[1-e^{-\frac{a_{3}}{1-\rho}} \sqrt{\frac{b_{3}}{\rho(1-\rho)}} K_{1}\left(\sqrt{\frac{b_{3}}{\rho(1-\rho)}}\right)\right]}_{J_{k, m}^{\mathrm{DOS}, \mathrm{FG}-\mathrm{AF}}(\rho)},
\end{aligned}
$$

where $a_{3}=\frac{\gamma_{\mathrm{th}} \lambda_{\mathrm{SR}_{k}}}{\bar{\gamma}}$ and $b_{3}=\frac{4\left(\bar{\gamma}+\lambda_{\mathrm{SR}_{k}}\right) \gamma_{\mathrm{th}^{2}} \lambda_{\mathrm{SR}_{k}} \lambda_{\mathrm{R}_{k} \mathrm{D}_{m}}}{\eta \bar{\gamma}^{2}}$.

The gradient of $P_{\text {out,minimizing }}^{\mathrm{DOS}, \mathrm{FG}-\mathrm{AF}}(\rho), \nabla P_{\mathrm{out}, \text { minimizing }}^{\mathrm{DOS}, \mathrm{FG}-\mathrm{AF}}(\rho)$, can be obtained as

$$
\begin{aligned}
\nabla & P_{\text {out }, \text { minimizing }}^{\mathrm{DOS}, \mathrm{FG}-\mathrm{AF}}(\rho) \\
& =\sum_{m=1}^{M} \sum_{k=1}^{K} \dot{J}_{k, m}^{\mathrm{DOS}, \mathrm{FG}-\mathrm{AF}}(\rho) \prod_{l=1, l \neq k}^{K} J_{l, m}^{\mathrm{DOS}, \mathrm{FG}-\mathrm{AF}}(\rho),
\end{aligned}
$$

where

$$
\begin{aligned}
& \dot{J}_{k, m}^{\mathrm{DOS}, \mathrm{FG}-\mathrm{AF}}(\rho) \\
& =\frac{e^{-\frac{a_{3}}{1-\rho}}}{2(1-\rho)^{2}}\left[2 a_{3} \sqrt{\frac{b_{3}}{\rho(1-\rho)} K_{1}}\left(\sqrt{\frac{b_{3}}{\rho(1-\rho)}}\right)\right. \\
& \left.\quad-\frac{b_{3}(1-2 \rho)}{\rho^{2}} K_{0}\left(\sqrt{\frac{b_{3}}{\rho(1-\rho)}}\right)\right]
\end{aligned}
$$

\section{DPS DF}

For the case of the DPS scheme using DF relaying, minimizing $P_{\text {out,approx }}^{\mathrm{DPS}, \mathrm{DF}}$ with respect to $\rho$ is equivalent to minimizing $P_{\text {out,minimizing }}^{\mathrm{DPS}, \mathrm{DF}}(\rho)$, which can be expressed as

$$
P_{\text {out,minimizing }}^{\mathrm{DPS}, \mathrm{DF}}(\rho)=1+\sum_{m=1}^{M} \sum_{k=1}^{K} \widetilde{\sum_{l}} J_{k, m}^{\mathrm{DPS}, \mathrm{DF}}(\rho) \text {, }
$$

where $J_{k, m}^{\mathrm{DPS}, \mathrm{DF}}(\rho)=J_{k, m}^{\mathrm{DOS}, \mathrm{DF}}(\rho)-1$ with $a_{1}=\frac{\gamma_{\mathrm{th}} \theta_{4}}{\bar{\gamma}}$, $b_{1}=\frac{\gamma_{\mathrm{th}} \lambda_{\mathrm{R}_{k} \mathrm{D}_{m}}}{\bar{\gamma} \eta}$, and $\theta_{4}=\widehat{\sum}_{t}$. Thus, the gradient of $P_{\text {out,minimizing }}^{\mathrm{DPS}, \mathrm{DF}}(\rho), \nabla P_{\text {out,minimizing }}^{\mathrm{DPS}, \mathrm{DF}}(\rho)$, can be expressed as

$$
\nabla P_{\text {out,minimizing }}^{\mathrm{DPS}, \mathrm{DF}}(\rho)=1+\sum_{m=1}^{M} \sum_{k=1}^{K} \widetilde{\sum_{l}} \dot{J}_{k, m}^{\mathrm{DPS}, \mathrm{DF}}(\rho),
$$

where $\dot{J}_{k, m}^{\mathrm{DPS}, \mathrm{DF}}(\rho)$ has a similar form to $\dot{J}_{k, m}^{\mathrm{DOS}, \mathrm{DF}}(\rho)$ as presented in (39).

\section{E. DPS VG-AF}

For the case of the DPS scheme using VG-AF relaying, minimizing $P_{\text {out,approx }}^{\mathrm{DPS}, \mathrm{VG}-\mathrm{AF}}$ with respect to $\rho$ is equivalent to minimizing $P_{\text {out,minimizing }}^{\mathrm{DPS}, \mathrm{G}-\mathrm{AF}}(\rho)$, which can be expressed as

$$
P_{\text {out,minimizing }}^{\mathrm{DPS}, \mathrm{VG}-\mathrm{AF}}(\rho)=\sum_{m=1}^{M} \sum_{k=1}^{K}\left[1+\widetilde{\sum_{l}} J_{k, m}^{\mathrm{DPS}, \mathrm{VG}-\mathrm{AF}}(\rho)\right] \text {, }
$$

where $J_{k, m}^{\mathrm{DPS}, \mathrm{VG}-\mathrm{AF}}(\rho)=J_{k, m}^{\mathrm{DOS}, \mathrm{VG}-\mathrm{AF}}(\rho)-1$ with $a_{2}=$ $\frac{\gamma_{\mathrm{th}} \theta_{5}}{\bar{\gamma}}, b_{2}=\frac{4 \gamma_{\mathrm{th}} \lambda_{\mathrm{R}_{k} \mathrm{D}_{m}} \theta_{5}}{\eta \bar{\gamma}}$, and $\theta_{5}=\widehat{\sum}_{t}$. Thus, the gradient of $P_{\text {out,minimizing }}^{\mathrm{DPS}, \mathrm{VG}-\mathrm{AF}}(\rho), \nabla P_{\text {out,minimizing }}^{\mathrm{DPS}, \mathrm{VG}-\mathrm{AF}}(\rho)$, can be expressed as

$$
\nabla P_{\text {out }, \text { minimizing }}^{\mathrm{DPS}, \mathrm{VG}-\mathrm{AF}}(\rho)=\sum_{m=1}^{M} \sum_{k=1}^{K} \widetilde{\sum_{l}} \dot{J}_{k, m}^{\mathrm{DPS}, \mathrm{VG}-\mathrm{AF}}(\rho),
$$

where $\dot{J}_{k, m}^{\mathrm{DPS}, \mathrm{VG}-\mathrm{AF}}(\rho)$ has a similar form to $\dot{J}_{k, m}^{\mathrm{DOS}, \mathrm{VG}-\mathrm{AF}}(\rho)$ as presented in (42).

\section{F. DPS FG-AF}

For the case of the DPS scheme using FG-AF relaying, minimizing $P_{\text {out.exact }}^{\mathrm{DPS}, \mathrm{AF}}$ with respect to $\rho$ is equivalent to minimizing $P_{\text {out,minimizing }}^{\mathrm{DPS}, \mathrm{FG}-\mathrm{AF}}(\rho)$, which can be expressed as

$$
P_{\text {out,minimizing }}^{\mathrm{DPS}, \mathrm{FG}-\mathrm{AF}}(\rho)=\sum_{m=1}^{M} \sum_{k=1}^{K}\left[1+\widetilde{\sum_{l}} J_{k, m}^{\mathrm{DPS}, \mathrm{FG}-\mathrm{AF}}(\rho)\right] \text {, }
$$

where $J_{k, m}^{\mathrm{DPS}, \mathrm{FG}-\mathrm{AF}}(\rho)=J_{k, m}^{\mathrm{DOS}, \mathrm{FG}-\mathrm{AF}}(\rho)-1$ with $a_{3}=$ $\frac{\gamma_{\mathrm{th}} \theta_{6}}{\bar{\gamma}}, b_{3}=\frac{4\left(\bar{\gamma}+\lambda_{\mathrm{SR}_{k}}\right) \gamma_{\mathrm{th}} \lambda_{\mathrm{R}_{k} \mathrm{D}_{m}} \theta_{6}}{\eta \bar{\gamma}^{2}}$, and $\theta_{6}=\widehat{\sum}_{t}$. Thus, the gradient of $P_{\text {out,minimizing }}^{\mathrm{DPS}, \mathrm{FG}-\mathrm{AF}}(\rho), \nabla P_{\text {out,minimizing }}^{\mathrm{DPS}, \mathrm{FG}-\mathrm{AF}}(\rho)$, can be expressed as

$$
\nabla P_{\text {out,minimizing }}^{\mathrm{DPS}, \mathrm{FG}-\mathrm{AF}}(\rho)=\sum_{m=1}^{M} \sum_{k=1}^{K} \widetilde{\sum_{l}} j_{k, m}^{\mathrm{DPS}, \mathrm{FG}-\mathrm{AF}}(\rho),
$$

where $\dot{J}_{k, m}^{\mathrm{DPS}, \mathrm{VG}-\mathrm{AF}}(\rho)$ has a similar form to $\dot{J}_{k, m}^{\mathrm{DOS}, \mathrm{VG}-\mathrm{AF}}(\rho)$ as presented in (42).

\section{Diversity Gain Analysis}

In oder to provide further insights into the obtained OPs, the diversity gain analysis of each selection scheme with different relaying strategies is carried out. The diversity gain ${ }^{9}$, $D$, achieved by a cooperative scheme can be defined as [41], [42]

$$
D=-\lim _{\bar{\gamma} \rightarrow \infty} \frac{\log P_{\text {out }}(\bar{\gamma})}{\log \bar{\gamma}}
$$

${ }^{9}$ It is noted that the diversity gain (order) only depends on the number of independent fading channels. In other words, the achievable diversity order is a function of the number of relays and destinations, but not the power-splitting ratio, in the considered multiuser multirelay cooperative network. 


\section{A. DOS Scheme}

1) DF Relaying Strategy: By using the fact that $e^{-\frac{1}{x}} \approx$ $1-\frac{1}{x}$ when $x \rightarrow \infty$, the asymptotic value of $\Omega$ in (66) can be given by $\Omega_{\mathrm{asym}}=\frac{1}{\bar{\gamma}^{M}} \prod_{m=1}^{M} \gamma_{\mathrm{th}} \lambda_{\mathrm{SD}_{m}}$. By utilizing the first two terms of (17), and then applying the following facts to (18), i.e., $\Gamma(0, x)=-\operatorname{Ei}(-x)$ [39, Eq. (8.359.1)], and $\operatorname{Ei}(-x) \approx C_{E}+\ln (x)$ when $x \rightarrow 0^{+}$[39, Eq. (8.214.1)], where $C_{E}$ denotes the Euler's constant [39, Eq. (8.367.1)], and after some algebraic manipulations, the asymptotic OP of the DOS scheme using DF strategy can be obtained as

$$
\begin{aligned}
& P_{\text {out,asym }}^{\mathrm{DOS}, \mathrm{DF}} \\
& =\left(\frac{1}{\bar{\gamma} M+K} \prod_{m=1}^{M} \gamma_{\mathrm{th}} \lambda_{\mathrm{SD}_{m}}\right) \sum_{m=1}^{M} \Theta_{m} \prod_{k=1}^{K}\left[\frac{\gamma_{\mathrm{th}_{\mathrm{h}}} \lambda_{\mathrm{SR}_{k}}}{1-\rho}\right. \\
& \left.\quad-\frac{\gamma_{\mathrm{th}} \lambda_{\mathrm{SR}_{k}} \lambda_{\mathrm{R}_{k} \mathrm{D}_{m}}}{\eta \rho}\left(C_{E}+\ln \left(\frac{\gamma_{\mathrm{th}} \lambda_{\mathrm{SR}_{k}}}{(1-\rho) \bar{\gamma}}\right)\right)\right] .
\end{aligned}
$$

Since $\lim _{x \rightarrow \infty} \frac{\log \left[\frac{1}{x^{M+K}}\left(c_{1}+\ln \left(\frac{c_{2}}{x}\right)\right)\right]}{\log x}=-(M+K)$, where $c_{1}, c_{2}$ are constants, it can be concluded that the diversity gain achieved by the DOS scheme using DF strategy is $M+K$.

2) VG-AF Strategy: Applying the formula $x K_{1}(x) \approx$ $1+\frac{x^{2}}{2} \ln \frac{x}{2}$ [24, Eq. (25)] to (24), and after some algebraic manipulations, the asymptotic OP of the DOS scheme using VG-AF strategy can be obtained as

$$
\begin{aligned}
P_{\text {out,asym }}^{\mathrm{DOS}, \mathrm{VG}-\mathrm{AF}} & \left(\frac{1}{\bar{\gamma}^{M+K}} \prod_{m=1}^{M} \gamma_{\mathrm{th}} \lambda_{\mathrm{SD}_{m}}\right) \sum_{m=1}^{M} \Theta_{m} \prod_{k=1}^{K}\left[\frac{\gamma_{\mathrm{th}} \lambda_{\mathrm{SR}_{k}}}{1-\rho}\right. \\
& -\frac{2 \gamma_{\mathrm{th}} \lambda_{\mathrm{SR}_{k}} \lambda_{\mathrm{R}_{k} \mathrm{D}_{m}}}{\eta \rho}\left(1-\frac{\gamma_{\mathrm{th}} \lambda_{\mathrm{SR}_{k}}}{(1-\rho) \bar{\gamma}}\right) \\
& \left.\times \ln \left(\sqrt{\frac{\gamma_{\mathrm{th}} \lambda_{\mathrm{SR}_{k}} \lambda_{\mathrm{R}_{k} \mathrm{D}_{m}}}{\eta \rho \gamma}}\right)\right]
\end{aligned}
$$

Since $\lim _{x \rightarrow \infty} \frac{\log \left[\frac{1}{x^{M+K}}\left[c_{1}+\left(c_{2}+\frac{c_{3}}{x}\right) \ln \left(\sqrt{\frac{c_{4}}{x}}\right)\right]\right]}{\log x}=-(M+K)$, where $c_{1}, \ldots, c_{4}$ are constants, it can be concluded that the diversity gain achieved by the DOS scheme using VG-AF strategy is $M+K$.

3) FG-AF Strategy: Using the similar method as in V-A2, the asymptotic OP of the DOS scheme using FG-AF strategy can be obtained as

$$
\begin{aligned}
& P_{\text {out }, \text { asym }}^{\mathrm{DOS}, \mathrm{FG}-\mathrm{AF}} \\
& =\left(\frac{1}{\bar{\gamma} M+K} \prod_{m=1}^{M} \gamma_{\mathrm{th}} \lambda_{\mathrm{SD}_{m}}\right) \sum_{m=1}^{M} \Theta_{m} \\
& \quad \times \prod_{k=1}^{K}\left[\frac{\gamma_{\mathrm{th}} \lambda_{\mathrm{SR}_{k}}}{1-\rho}-\left(1-\frac{\gamma_{\mathrm{th}} \lambda_{\mathrm{SR}_{k}}}{(1-\rho) \bar{\gamma}}\right)\right. \\
& \quad \times\left(\frac{2 \gamma_{\mathrm{th}} \lambda_{\mathrm{SR}_{k}} \lambda_{\mathrm{R}_{k} \mathrm{D}_{m}}}{\eta \rho(1-\rho)}+\frac{2 \gamma_{\mathrm{th}} \lambda_{\mathrm{SR}_{k}}^{2} \lambda_{\mathrm{R}_{k} \mathrm{D}_{m}}}{\eta \rho(1-\rho) \bar{\gamma}}\right) \\
& \left.\quad \times \ln \left(\sqrt{\frac{\gamma_{\mathrm{th}}\left(\bar{\gamma}+\lambda_{\mathrm{SR}_{k}}\right) \lambda_{\mathrm{SR}_{k}} \lambda_{\mathrm{R}_{k} \mathrm{D}_{m}}}{\eta \rho(1-\rho) \bar{\gamma}^{2}}}\right)\right] .
\end{aligned}
$$

Since $\lim _{x \rightarrow \infty} \frac{\log \left[\frac{1}{x^{M}+K}\left[c_{1}+\left(c_{2}+\frac{c_{3}}{x}+\frac{c_{4}}{x^{2}}\right) \ln \left(\sqrt{\frac{c_{5}}{x}}\right)\right]\right]}{\log x}=-(M+$ $K)$, where $c_{1}, \ldots, c_{5}$ are constants, it can be concluded that the diversity gain achieved by the DPS scheme using FG-AF strategy is $M+K$.

\section{B. DPS Scheme}

Using the similar method as done in $\mathrm{V}-\mathrm{A}$, the asymptotic OP of the DPS scheme using DF, VG-AF, and FG-AF strategies can be, respectively, obtained as

$$
\begin{aligned}
& P_{\text {out,asym }}^{\text {DPS,DF }} \\
& =\left(\frac{1}{\bar{\gamma}^{M+1}} \prod_{m=1}^{M} \gamma_{\mathrm{th} \lambda_{\mathrm{SD}_{m}}}\right) \sum_{m=1}^{M} \Theta_{m} \sum_{k=1}^{K} \Xi_{k} \widetilde{\sum_{l}} \\
& \times\left[\frac{\gamma_{\mathrm{th}} \widehat{\sum}_{t}}{1-\rho}-\frac{\gamma_{\mathrm{th}} \lambda_{\mathrm{R}_{k} \mathrm{D}_{m}} \widehat{\sum}_{t}}{\eta \rho}\left[C_{E}+\ln \left(\frac{\gamma_{\mathrm{th}} \widehat{\sum}_{t}}{(1-\rho) \bar{\gamma}}\right)\right]\right], \\
& P_{\text {out,asym }}^{\mathrm{DPS}, \mathrm{VG}-\mathrm{AF}} \\
& =\left(\frac{1}{\bar{\gamma}^{M+1}} \prod_{m=1}^{M} \gamma_{\mathrm{th}} \lambda_{\mathrm{SD}_{m}}\right) \sum_{m=1}^{M} \Theta_{m} \sum_{k=1}^{K} \Xi_{k} \widetilde{\sum_{l}} \\
& \times\left[\frac{\gamma_{\mathrm{th}} \widehat{\sum}_{t}}{1-\rho}-\frac{2 \gamma_{\mathrm{th}} \lambda_{\mathrm{R}_{k} \mathrm{D}_{m}} \widehat{\sum}_{t}}{\eta \rho}\left(1-\frac{\gamma_{\mathrm{th}} \widehat{\sum}_{t}}{(1-\rho) \bar{\gamma}}\right)\right. \\
& \left.\times \ln \left(\sqrt{\frac{\gamma_{\mathrm{th}} \lambda_{\mathrm{R}_{k} \mathrm{D}_{m}} \widehat{\sum}_{t}}{\eta \rho \bar{\gamma}}}\right)\right], \\
& P_{\text {out,asym }}^{\mathrm{DPS}, \mathrm{FG}-\mathrm{AF}} \\
& =\left(\frac{1}{\bar{\gamma}^{M+1}} \prod_{m=1}^{M} \gamma_{\mathrm{th}_{\mathrm{h}}} \lambda_{\mathrm{SD}_{m}}\right) \sum_{m=1}^{M} \Theta_{m} \sum_{k=1}^{K} \Xi_{k} \widetilde{\sum_{l}} \\
& \times\left[\frac{\gamma_{\mathrm{th}} \widehat{\sum}_{t}}{1-\rho}-\left(1-\frac{\gamma_{\mathrm{th}} \widehat{\sum}_{t}}{(1-\rho) \bar{\gamma}}\right)\right. \\
& \times\left(\frac{2 \gamma_{\mathrm{th}_{\mathrm{h}}} \lambda_{\mathrm{R}_{k} \mathrm{D}_{m}} \widehat{\sum}_{t}}{\eta \rho(1-\rho)}+\frac{2 \gamma_{\mathrm{th}_{\mathrm{h}}} \lambda_{\mathrm{SR}_{k}} \lambda_{\mathrm{R}_{k} \mathrm{D}_{m}} \widehat{\sum}_{t}}{\eta \rho(1-\rho) \bar{\gamma}}\right) \\
& \times \ln \left(\sqrt{\left.\frac{\gamma_{\mathrm{th}}\left(\bar{\gamma}+\lambda_{\mathrm{SR}_{k}}\right) \lambda_{\mathrm{R}_{k} \mathrm{D}_{m} \widehat{\sum}_{t}}}{\eta \rho(1-\rho) \bar{\gamma}^{2}}\right)} .\right.
\end{aligned}
$$

With similar arguments used in V-A, it is straightforward that the diversity gain achieved by the DOS scheme using either $\mathrm{DF}, \mathrm{VG}-\mathrm{AF}$, or FG-AF strategies is $M+1$.

\section{NumericAl RESUlts AND Discussions}

In this section, representative numerical examples are provided to illustrate the outage performance of the DPS and DOS schemes. Insightful discussions related to the impact of EH process on the system performance will be presented. Monte-Carlo simulation results are provided to validate the developed analysis. In simulation setting, we assume that the source, the relays (with the same coordinates), and the destinations (with the same coordinates) form a line network, which is well used in the literature [25], [29]. We set: $M=3$, $K=3$, the distance between the source and the destinations $d_{\mathrm{SD}}=10 \mathrm{~m}$, the distance between the source and the relays $d_{\mathrm{SR}}=3 \mathrm{~m}$, the distance between the relays and the destinations $d_{\mathrm{RD}}=d_{\mathrm{SD}}-d_{\mathrm{SR}}$, the reference distance $d_{0}=1$ 


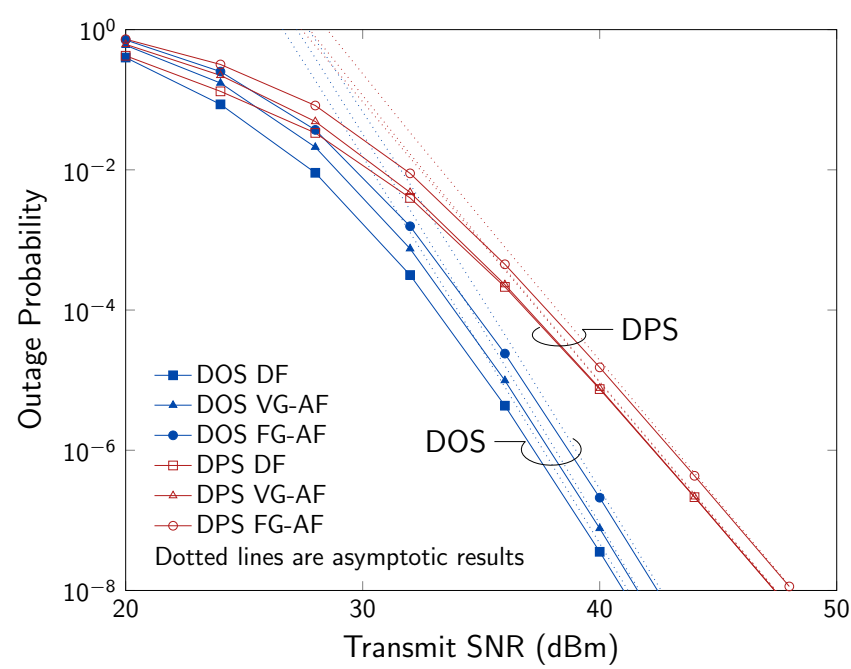

Fig. 3. Outage probability of the DOS and DPS schemes as a function of transmit SNR $\bar{\gamma}(\mathrm{dBm})$ with $\rho=0.5$. Performance curves are generated using exact results.

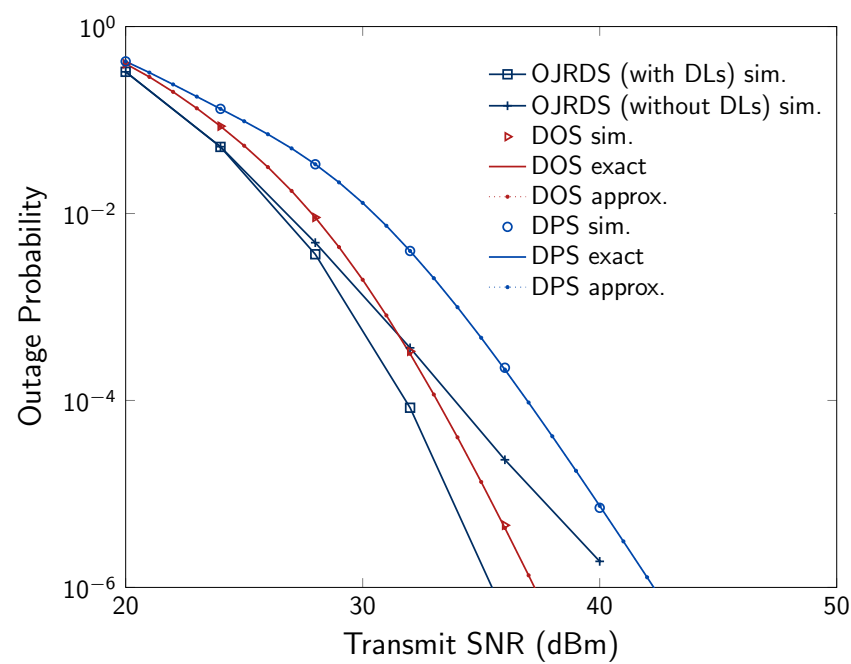

Fig. 4. Performance comparison among the OJRDS with and without direct links (DLs), DOS, and DPS schemes using DF relaying strategy with $\rho=0.5$.

$\mathrm{m}$, and the path-loss at $d_{0}$ is $\mathcal{L}=-30 \mathrm{~dB}$, the path-loss exponent $\epsilon=2.7$, the energy conversion efficiency of the EH process $\eta=0.7$ (as mentioned ealier, a fixed $\eta$ has been well assumed in the literature [23], [33], [36]), and the fixed target data rate $R_{\mathrm{th}}=1 \mathrm{bit} / \mathrm{s} / \mathrm{Hz}$. In the optimal analysis of $\rho$, we set step-size $s_{z}=10^{-2}$, stopping threshold $s_{t}=10^{-6}$. The first 10 terms of the Maclaurin series in (17) are used to generate curves for approximate values in the case of using DF strategy.

The performance of the optimal joint relay-destination selection (OJRDS) scheme is used as a benchmark. Mathematically, the selection criterion of the OJRDS scheme can be written as on the top of the next page.

To the best of authors' knowledge, such max-max selection criterion has not been investigated in the literature since its performance analysis is intractable. It is evident from (59)(61) that the OJRDS scheme requires a high computational complexity. Therefore, this will be the main purpose of the proposal of our schemes is to reduce the computational complexity, yet provide comparable performance gain and diversity gain, compared to the optimal scheme.

Fig. 3 shows the outage probability (OP) of the DOS and DPS schemes as a function of the transmit SNR for the three relaying strategies, i.e., DF, VG-AF, and FG-AF. Considering the DOS scheme, the OP achieved by the DF, VG-AF, and FG$\mathrm{AF}$ strategies increases with the mentioned order. It means that the DF strategy gives the best performance while the FG-AF strategy gives the worst performance. Additionally, employing FG-AF in SWIPT networks raises some disadvantages, such as maintaining a fixed gain is likely difficult since the transmission power of relays depends on the instantaneous harvested energy. However, our aim is to make the analysis as much general as possible so that it could encompass different kinds of relaying strategies. Also, please note that FG-AF strategy can be employed in static or low-mobility networks, where the harvested energy is likely stable due to the quasi-static wireless environments. Since the harvested energy does not much fluctuate, a relay can adjust its fixed gain based on the statistical record of harvested energy.

Also, from Fig. 3, the DPS scheme has the same behavior as of the DOS scheme in the low SNR regime. But at the high SNR regime, the performances of the DF and VG-AF schemes are very similar. On the other hand, based on the slope of performance curves at high SNR regime, we can observe that, using the same relaying strategy, the DOS scheme achieved higher diversity gain than the DPS scheme does.

Fig. 4 presents the performance comparison among the OJRDS (with and without direct links), DOS and DPS schemes. As can be seen, for a given value of SNR and a specific relaying strategy, performance of the OJRDS scheme with direct links is the best among that of all selection schemes. While performance of the OJRDS scheme without direct links is better than of DOS and DPS scheme in low SNR regime but it tends to be vice versa in high SNR regime. According to our study, the performance curves of different relaying strategies have the same behavior (trend). Thus, we only show the results for the case of the DF strategy for brevity and avoid repetition.

Fig. 4 shows the diversity behavior of the DOS scheme and the DPS scheme using the DF relaying strategy, respectively. According to our study, the performance curves of different relaying strategies have the same behavior (trend). Thus, we only show the results for the case of the DF strategy for brevity and avoid repetition. Recall that the diversity gain can be indicated by the slope at high SNR regions of an outage performance curve [25], [42].

As shown in Fig. 5a, for a given $M$ (or $K$ ), the slope of the curves varies as $K$ (or $M$ ) increases. In addition, performance curves for the case of there are $M$ destinations and $K$ relays are parallel to the curve for $1 / \bar{\gamma}^{M+K}$ at high SNR regime, which results in the diversity gain of the DOS scheme equals to $M+K$. Thus, we can conclude that by combining direct links and opportunistic relay selection protocol, full diversity gain can still be obtained in SWIPT cooperative networks.

As can be observed in Fig. 5b, for a given number of 


$$
\left(\mathrm{R}_{\mathrm{b}}, \mathrm{D}_{\mathrm{b}}\right)=\arg \max _{1 \leq m \leq M} \max \left\{\left|h_{\mathrm{SD}_{m}}\right|^{2}, \max _{1 \leq k \leq K} \min \left\{(1-\rho) \bar{\gamma}\left|h_{\mathrm{SR}_{k}}\right|^{2}, \eta \rho \bar{\gamma}\left|h_{\mathrm{SR}_{k}}\right|^{2}\left|h_{\mathrm{R}_{k} \mathrm{D}_{\mathrm{b}}}\right|^{2}\right\}\right\}
$$

for the case of using DF relaying strategy, and

$$
\left(\mathrm{R}_{\mathrm{b}}, \mathrm{D}_{\mathrm{b}}\right)=\arg \max _{1 \leq m \leq M} \max \left\{\left|h_{\mathrm{SD}_{m}}\right|^{2}, \max _{1 \leq k \leq K}\left\{\frac{\eta \rho(1-\rho) \bar{\gamma}^{2}\left|h_{\mathrm{SR}_{k}}\right|^{4}\left|h_{\mathrm{R}_{k} \mathrm{D}_{\mathrm{b}}}\right|^{2}}{(1-\rho) \bar{\gamma}\left|h_{\mathrm{SR}_{k}}\right|^{2}+\eta \rho \bar{\gamma}\left|h_{\mathrm{SR}_{k}}\right|^{2}\left|h_{\mathrm{R}_{k} \mathrm{D}_{\mathrm{b}}}\right|^{2}+1}\right\}\right\}
$$

for the case of using VG-AF relaying strategy, and

$$
\left(\mathrm{R}_{\mathrm{b}}, \mathrm{D}_{\mathrm{b}}\right)=\arg \max _{1 \leq m \leq M} \max \left\{\left|h_{\mathrm{SD}_{m}}\right|^{2}, \max _{k}\left\{\frac{\eta \rho(1-\rho) \bar{\gamma}^{2}\left|h_{\mathrm{SR}_{k}}\right|^{4}\left|h_{\mathrm{R}_{k} \mathrm{D}_{\mathrm{b}}}\right|^{2}}{(1-\rho) \kappa_{k}\left|h_{\mathrm{SR}_{k}}\right|^{2}+\eta \rho \bar{\gamma}\left|h_{\mathrm{SR}_{k}}\right|^{2}\left|h_{\mathrm{R}_{k} \mathrm{D}_{\mathrm{b}}}\right|^{2}}\right\}\right\}
$$

for the case of using FG-AF relaying strategy.

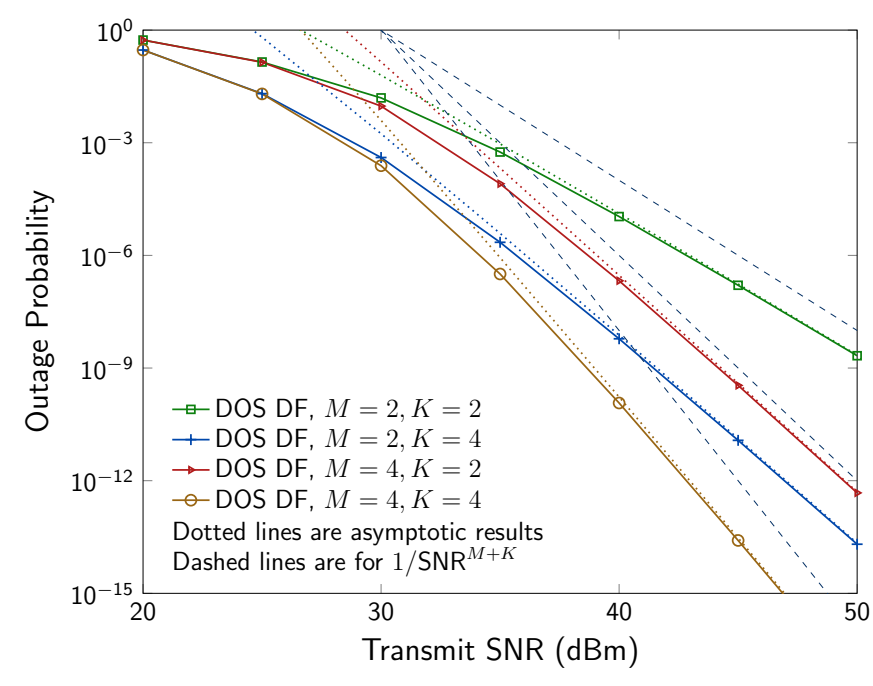

(a) DOS scheme using DF strategy

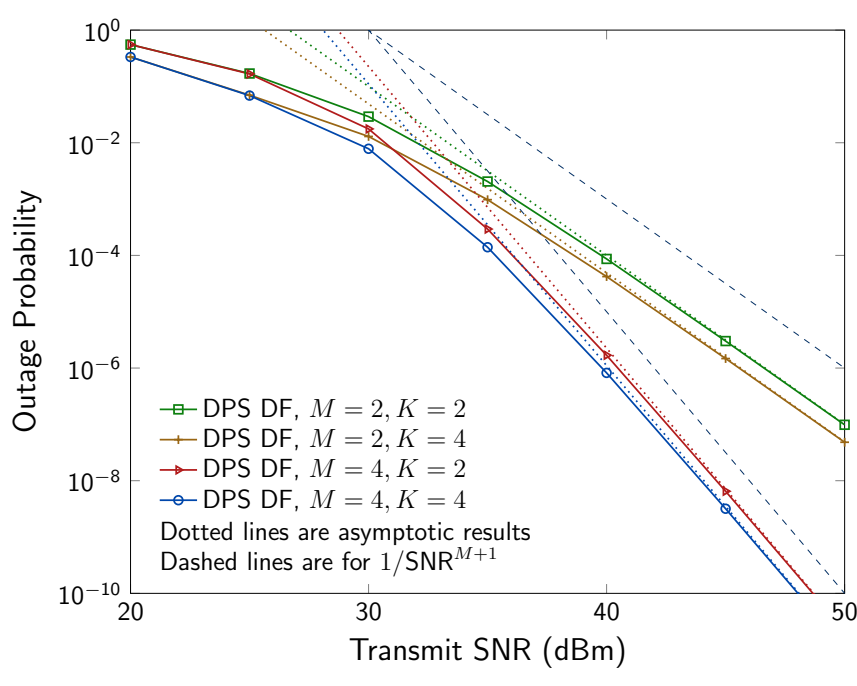

(b) DPS scheme using DF strategy

Fig. 5. Diversity gain of a) the DOS scheme and b) the DPS scheme using DF relaying strategy with different numbers of relays and destinations, $\rho=0.5$. Performance curves are generated using exact results.

destinations, $M$, as the number of relays, $K$, increases, the slope of the curves is the same, which applies in non-diversity gain. In contrast, for a given $K$, the slope of the curves varies as $M$ increases. On the other hand, regardless of $K$, performance curves for the case of there are $M$ destinations are parallel to the curve for $1 / \bar{\gamma}^{M+1}$ at high SNR regime, which results in the diversity gain of the DPS scheme equals to $M+1$.

Fig. 6 plots the OP of the DPS and DOS schemes as a function of the power splitting ratio $\rho$. As can be observed, for a given value of $\rho$, a certain relaying strategy, e.g., DF, VG-AF, or FG-AF, in the DOS scheme outperforms its counterpart in the DPS scheme, which is in line with the observation in Fig. 3. On the other hand, in each scheme, the OP increases in the order of DF, VG-AF, and FG-AF strategies. It is noteworthy that any OP appears as a convex function with respect to $\rho \in$ $(0,1)$. Consequently, there is an optimal value of $\rho$ where the $\mathrm{OP}$ is minimum as illustrated in Fig. 6. Note that the minimum OPs are obtained using the proposed gradient-based search method.

Fig. 7 shows the optimal value of the power splitting ratio $\rho^{*}$, obtained using the proposed search method, of each relaying strategy for both DPS and DOS schemes. In other

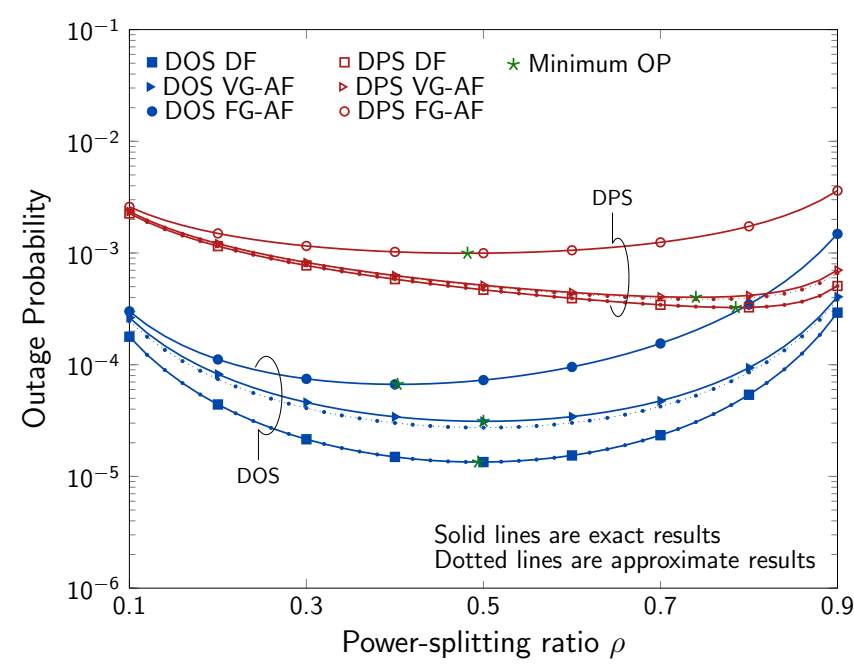

Fig. 6. Outage probability as a function of the power splitting ratio $\rho$ with $\bar{\gamma}=35 \mathrm{dBm}$

words, $\rho^{*}$ indicates the optimal portion of received power that should be fed to the harvesting energy module in order to 


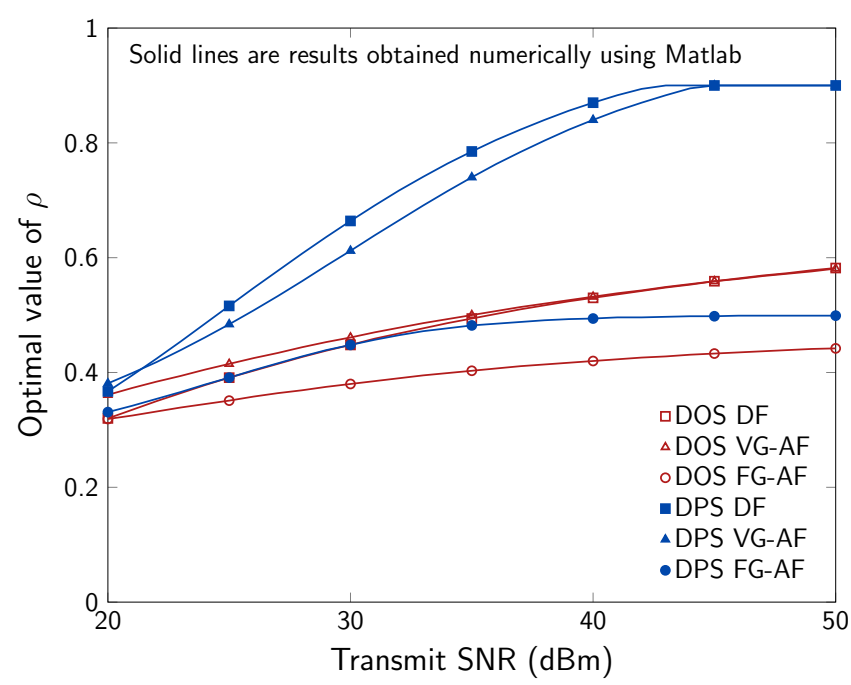

Fig. 7. Optimal value of the power splitting ratio $\rho^{*}$ (obtained using the proposed search method) as a function of transmit SNR (dBm).

achieve the best outage performance. As can be observed in Fig. 7, in all scenarios, when the source increases its transmit power, the relay needs to increase the harvested energy in order to achieve the minimum OP. In the DPS scheme, the $\rho^{*}$ used for FG-AF, VG-AF, and DF strategies increases with the mentioned order. In the DOS scheme, $\rho^{*}$ used for VG-AF and DF strategies are similar and higher than that used for FG-AF strategy. Generally, the DPS scheme needs greater harvested energy than the DOS scheme in order to achieve their own minimum OP.

Next, we discuss more about the role of direct links in the considered selection schemes. Fig. 8 plots OPs of OJRDS scheme (with and without directs links), DOS scheme, and DPS scheme as a function of the distance between source and relays, $d_{\mathrm{SR}}$. As can be observed, performance of the OJRDS (without direct links) become worse than that of DOS and DPS schemes as the source-relay distance is larger. In addition, the OPs of OJRDS (with direct links), DOS, and DPS schemes increase as $d_{\mathrm{SR}}$ increases, and become similar at high value of $d_{\mathrm{SR}}$. One possible reason is that when the selected relay is located far away from the source, the relaying transmission depending on the harvested energy is dominated by the direct transmission.

The complexity level of a selection scheme can be defined in terms of the number of channels that require CSI estimation, denoted by $N_{\mathrm{csi}}$, and the number of potential channels for comparisons to find out the selected relay-destination pair, denoted by $N_{\text {com }}$. Please note that the relay-destination process is consisted of steps. Firstly, it conducts CSI estimation of the considered channels. Then, it compares the acquired channel conditions, i.e., CSI, to figure out what the best channel is. The values of $N_{\mathrm{csi}}$ and $N_{\mathrm{com}}$ are summarized in Table. 1, in which their units concern to the number of channels. For instance, for a large-scale multiuser multirelay cooperative network with $M=K=10$, the required amounts of CSI estimations are 21,30 , and 120 for the DPS scheme, the DOS scheme, and

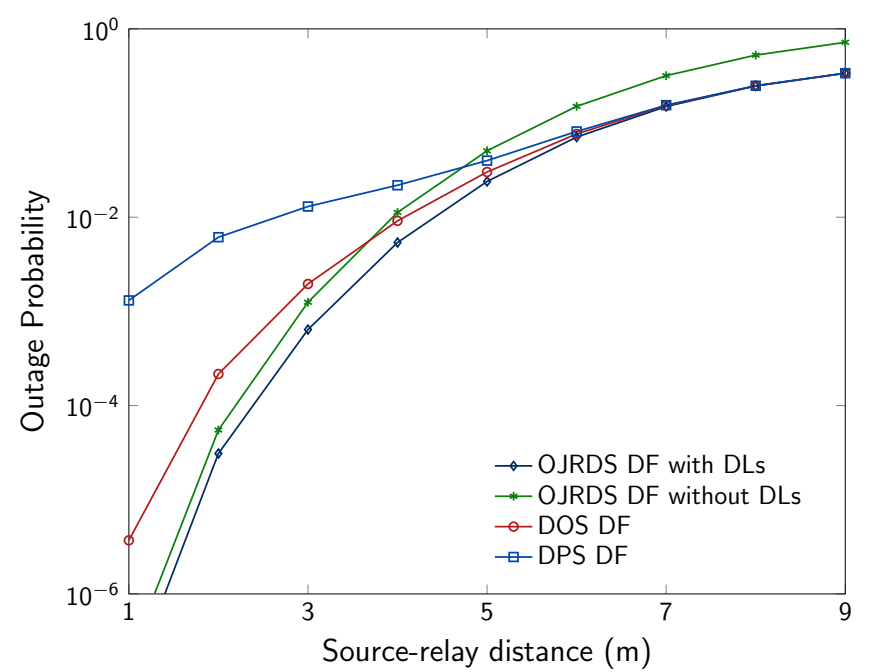

Fig. 8. Outage probability of the DOS DF case as a function of distance between source and relays with $\bar{\gamma}=30 \mathrm{dBm}, \rho=0.5$. Performance curves are generated using simulation results.

the OJRDS scheme, respectively. In addition, the number of comparisons of potential links for the proposed schemes and the OJRDS scheme are 30 and 210 , perspectively, in the case of using DF strategy, while they are 20 and 110, respectively, in the case of using AF strategies. Thus, it is clear that the complexity of the proposed schemes is much lower than that of the OJRDS scheme.

\section{CONCLUSIONS}

In this paper, studying the opportunistic scheduling for downlink scenarios in multiuser multirelay cooperative networks with SWIPT employed at relays, two low-complexity, yet efficient, relay-destination selection schemes, namely the DOS scheme and the DPS scheme, have been proposed by exploiting the direct links between the source and the destinations. Three well-known relaying strategies, i.e, DF, VG-AF, and FG-AF, have been respectively adopted in each proposed scheme and the corresponding performance of each scenario has been analyzed in terms of outage probability (OP). In particular, analytical expressions and closed-form approximate expressions for the OP of the DF and VG-AF strategies, and closed-form expressions for the OP of the FG-AF strategy in the both schemes have been derived and corroborated through Monte-Carlo simulations. Moreover, the gradient-based search method has been proposed to find out the optimal values of $\rho^{*}$ that minimize the attained OPs. For a given value of SNR and a specific relaying strategy, DOS scheme outperforms DPS scheme. On the other hand, DOS scheme achieves the diversity gain of $M+K$, which is higher than that achieved by DPS scheme, which is $M+1$. In both DPS scheme and DOS scheme, outage performance of DF is superior to that of VG-AF and FG-AF. 
TABLE I

Summarizing the Main Characteristics of the Considered Relay-Destination Selection Schemes

\begin{tabular}{llll}
\hline & OJRDS & DOS & DPS \\
\hline Diversity order (DF and AF) & $M+K$ & $M+K$ & $M+1$ \\
Number of required CSI estimations, $N_{\mathrm{csi}},(\mathrm{DF}$ and AF) & $M+K+K M$ & $M+2 K$ & $M+K+1$ \\
Number of comparisons of potential links, $N_{\text {com }}(\mathrm{DF}$ only) & $M+2 K M$ & $M+2 K$ & $M+2 K$ \\
Number of comparisons of potential links, $N_{\mathrm{com}}(\mathrm{AF}$ only) & $M+K M$ & $M+K$ & $M+K$ \\
\hline
\end{tabular}

\section{APPENDIX A}

PROOF OF THEOREM 1

Since all wireless links are assumed to be independent, using the total probability theorem [43], (15) can be expressed as

$$
\begin{aligned}
P_{\text {out }}^{\mathrm{DOS}, \mathrm{DF}} & \\
= & \underbrace{\operatorname{Pr}\left(\gamma_{\mathrm{SD}_{\mathrm{b}}}<\gamma_{\mathrm{th}}\right)}_{\Omega} \sum_{m=1}^{M} \underbrace{\operatorname{Pr}\left(\mathrm{D}_{\mathrm{b}}=\mathrm{D}_{m}\right)}_{\Theta_{m}} \\
& \times \prod_{k=1}^{K} \underbrace{\operatorname{Pr}\left(\min \left\{\alpha\left|h_{\mathrm{SR}_{k}}\right|^{2}, \beta\left|h_{\mathrm{SR}_{k}}\right|^{2}\left|h_{\mathrm{R}_{k} \mathrm{D}_{m}}\right|^{2}\right\}<\gamma_{\mathrm{th}}\right)}_{\mathrm{A}} .
\end{aligned}
$$

The following lemma helps reader easily follow how to obtain $\operatorname{Pr}\left(\gamma_{\mathrm{SD}_{\mathrm{b}}}<\gamma_{\mathrm{th}}\right)$.

Lemma 3: Let $\left|h_{\mathrm{b}}\right|^{2} \triangleq \max _{1 \leq l \leq L}\left\{\left|h_{l}\right|^{2}\right\}, \quad l \in$ $\{1,2, \ldots, L\}$, where $\left|h_{\mathrm{b}}\right|^{2} \in\left\{\left|h_{\mathrm{SD}_{\mathrm{b}}}\right|^{2},\left|h_{\mathrm{SR}_{\mathrm{b}}}\right|^{2}\right\},\left|h_{l}\right|^{2} \in$ $\left\{\left|h_{\mathrm{SD}_{m}}\right|^{2},\left|h_{\mathrm{SR}_{k}}\right|^{2}\right\}, L \in\{M, K\}$. The cumulative distribution function (CDF), $F_{\left|h_{\mathrm{b}}\right|^{2}}(z)$, and the probability density function (PDF), $f_{\left|h_{\mathrm{b}}\right|^{2}}(z)$, of $\left|h_{\mathrm{b}}\right|^{2}$ can be given by

$$
\begin{aligned}
& F_{\left|h_{\mathrm{b}}\right|^{2}}(z)=1+\sum_{l=1}^{L}(-1)^{l} \sum_{\substack{q_{1}=1 \\
q_{1}<\cdots<q_{l}}}^{L} \cdots \sum_{\substack{q_{l}=1 \\
q_{1}}}^{L} e^{-z \sum_{t=1}^{l} \lambda_{q_{t}}} \\
& f_{\left|h_{\mathrm{b}}\right|^{2}}(z)=\sum_{l=1}^{L}(-1)^{l+1} \sum_{\substack{q_{1}=1 \\
q_{1}<\cdots<q_{l}}}^{L} \cdots \sum_{\substack{q_{l}=1 \\
q_{1}}}^{L}\left(\sum_{t=1}^{l} \lambda_{q_{t}}\right) e^{-z \sum_{t=1}^{l} \lambda_{q_{t}}} .
\end{aligned}
$$

Proof: Since $\left|h_{l}\right|^{2}$,s are independent random variables, the CDF of $\left|h_{\mathrm{b}}\right|^{2}$ can be given by $F_{\left|h_{\mathrm{b}}\right|^{2}}(z)=$ $\prod_{l=1}^{L} \operatorname{Pr}\left(\left|h_{l}\right|^{2}<z\right)$. By using the following the multinomial expansion identity

$$
\prod_{l=1}^{L}\left(1-x_{l}\right)=\sum_{l=0}^{L}(-1)^{l} \sum_{\substack{q_{1}=1 \\ q_{1}<\cdots<q_{l}}}^{L} \cdots \sum_{\substack{q_{l}=1 \\ q^{\prime}}}^{L} \prod_{t=1}^{l} x_{q_{t}},
$$

and after some algebraic steps, $F_{\left|h_{\mathrm{b}}\right|^{2}}$ can be obtained as in (63). By taking derivative of the term in the right hand side of (63), the PDF of $\left|h_{\mathrm{b}}\right|^{2}$ can be obtained as in (64). This completes the proof of Lemma 3.
Using (7) and invoking Lemma 3, the probability $\operatorname{Pr}\left(\gamma_{\mathrm{SD}_{\mathrm{b}}}<\gamma_{\mathrm{th}}\right)$ can be given by

$$
\Omega=1+\sum_{l=1}^{M}(-1)^{l} \sum_{\substack{q_{1}=1 \\ q_{1}<\cdots<q_{l}}}^{M} \cdots \sum_{\substack{q_{l}=1 \\ M}}^{M} e^{-\frac{\gamma_{\text {th }}}{\bar{\gamma}} \sum_{t=1}^{l} \lambda_{\mathrm{SD}_{q_{t}}} .}
$$

Next, $\Theta_{m}$ in (62) is derived in the following lemma.

Lemma 4: Let $\Theta_{m} \triangleq \operatorname{Pr}\left(\mathrm{D}_{\mathrm{b}}=\mathrm{D}_{m}\right)$ and $\Xi_{k} \triangleq \operatorname{Pr}\left(\mathrm{R}_{\mathrm{b}}=\right.$ $\mathrm{R}_{k}$ ) (which will be used for the DPS case). $\Theta_{m}$ and $\Xi_{k}$ can be, respectively, given by

$$
\begin{gathered}
\Theta_{m}=1+\sum_{\substack{l=1 \\
l \neq m}}^{M}(-1)^{l} \sum_{\substack{q_{1}=1, \neq m \\
q_{1}<\cdots<q_{l}}}^{M} \cdots \sum_{\substack{q_{l}=1, \neq m \\
\mathrm{SD}_{m}}}^{M} \frac{\lambda_{\mathrm{SD}_{m}}}{\lambda_{t=1} \lambda_{\mathrm{SD}_{q_{t}}}}, \\
\Xi_{k}=1+\sum_{\substack{l=1 \\
l \neq k}}^{K}(-1)^{l} \sum_{\substack{q_{1}=1, \neq k \\
q_{1}<\cdots<q_{l}}}^{K} \cdots \sum_{\substack{q_{l}=1, \neq k \\
\mathrm{SR}_{k}}}^{K} \frac{\lambda_{\mathrm{SR}_{k}}}{\sum_{t=1} \lambda_{\mathrm{SR}_{q_{t}}}} .
\end{gathered}
$$

Proof: Invoking a similar method presented in [44] and making use of the multinomial expansion identity in (65), (67) and (68) can be easily obtained. This completes the proof of Lemma 4.

We are going to work on the remaining part of (62) as follows. Let $Y \triangleq\left|h_{\mathrm{SR}_{k}}\right|^{2}$ and conditioning on $Y=y$, A can be further expressed as

$$
\mathrm{A}=\int_{0}^{\infty}\left[1-\operatorname{Pr}\left(\alpha y \geq \gamma_{\mathrm{th}}\right) \operatorname{Pr}\left(\beta y\left|h_{\mathrm{R}_{k} \mathrm{D}_{m}}\right|^{2} \geq \gamma_{\mathrm{th}}\right)\right] f_{Y}(y) d y .
$$

where $f_{Y}(y)$ denotes the PDF of $Y$. Since $Y=\left|h_{\mathrm{SR}_{k}}\right|^{2}$ and $\left|h_{\mathrm{R}_{k} \mathrm{D}_{m}}\right|^{2}$ follow exponential distributions with rate parameters $\lambda_{\mathrm{SR}_{k}}$ and $\lambda_{\mathrm{R}_{k} \mathrm{D}_{m}}$, respectively, $\mathrm{A}$ can be obtained as $\mathrm{A}=$

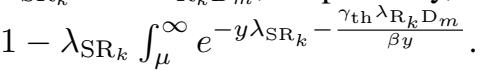

By plugging (69) and (66) into (62), and invoking Lemma 4, (16) can be obtained. This completes the proof of Theorem 1.

\section{APPENDIX B}

\section{PROOF OF THEOREM 2}

Since all wireless links are assumed to be independent, using the total probability theorem [43], $P_{\mathrm{out}}^{\mathrm{DOS}, \mathrm{VG}-\mathrm{AF}}$ in (19) 
can be expressed as

$$
\begin{aligned}
P_{\text {out }}^{\text {DOS,VG-AF }} & \\
= & \underbrace{\operatorname{Pr}\left(\gamma_{\mathrm{SD}_{\mathrm{b}}}<\gamma_{\mathrm{th}}\right)}_{\Omega} \sum_{m=1}^{M} \underbrace{\operatorname{Pr}\left(\mathrm{D}_{\mathrm{b}}=\mathrm{D}_{m}\right)}_{\Theta_{m}} \\
& \times \prod_{k=1}^{K} \underbrace{\operatorname{Pr}\left(\frac{\alpha\left|h_{\mathrm{SR}_{k}}\right|^{2} \beta\left|h_{\mathrm{SR}_{k}}\right|^{2}\left|h_{\mathrm{R}_{k} \mathrm{D}_{m}}\right|^{2}}{\alpha\left|h_{\mathrm{SR}_{k}}\right|^{2}+\beta\left|h_{\mathrm{SR}_{k}}\right|^{2}\left|h_{\mathrm{R}_{k} \mathrm{D}_{m}}\right|^{2}+1}<\gamma_{\mathrm{th}}\right)}_{\mathrm{B}} .
\end{aligned}
$$

Next, conditioning on $\left|h_{\mathrm{SR}_{k}}\right|^{2}=x$, B in (70) can be rewritten as

$$
\begin{aligned}
\mathrm{B}= & \int_{0}^{\infty} \operatorname{Pr}\left(\left(\alpha x-\gamma_{\mathrm{th}}\right) \beta x\left|h_{\mathrm{R}_{k} \mathrm{D}_{m}}\right|^{2}<\alpha \gamma_{\mathrm{th}} x+\gamma_{\mathrm{th}}\right) \\
& \times f_{\left|h_{\mathrm{SR}_{k}}\right|^{2}}(x) d x
\end{aligned}
$$

As can be seen, if $x \in\left[0, \gamma_{\mathrm{th}} / \alpha\right]$, the probability in (71) is always 1. Thus, (71) can be written as

$$
\begin{aligned}
\mathrm{B}= & \int_{0}^{\mu} f_{X}(x) d x \\
& +\int_{\mu}^{\infty} \operatorname{Pr}\left(\left|h_{\mathrm{R}_{k} \mathrm{D}_{m}}\right|^{2}<\frac{\alpha \gamma_{\mathrm{th}} x+\gamma_{\mathrm{th}}}{\left(\alpha x-\gamma_{\mathrm{th}}\right) \beta x}\right) f_{X}(x) d x .
\end{aligned}
$$

where $\mu=\gamma_{\mathrm{th}} / \alpha$. Since $\left|h_{\mathrm{SR}_{k}}\right|^{2}$ and $\left|h_{\mathrm{R}_{k} \mathrm{D}_{m}}\right|^{2}$ follow the exponential distributions with rate parameters $\lambda_{\mathrm{SR}_{k}}$ and $\lambda_{\mathrm{R}_{k} \mathrm{D}_{m}}$, respectively, after some algebraic manipulations, $\mathrm{B}$ can be given by

$$
\mathrm{B}=1-\lambda_{\mathrm{SR}_{k}} \int_{\mu}^{\infty} e^{-y \lambda_{\mathrm{SR}_{k}}-\frac{\left(\alpha \gamma_{\mathrm{th}} y+\gamma_{\mathrm{th}}\right) \lambda_{\mathrm{R}_{k} \mathrm{D}_{m}}}{\left(\alpha y-\gamma_{\mathrm{th}}\right) \beta y}} d y
$$

Plugging (66), (67), and (73) into (70), (20) can be obtained. This completes the proof of Theorem 2 .

\section{APPENDIX C \\ ProOF OF THEOREM 3}

Since all wireless links are assumed to be independent, using the total probability theorem [43], $P_{\text {out,exact }}^{\mathrm{DOS}, \mathrm{AF}}$ in $(25)$ can be expressed as

$$
\begin{aligned}
P_{\text {out,exact }}^{\mathrm{DOS}, \mathrm{AG}} & \\
= & \underbrace{\operatorname{Pr}\left(\gamma_{\mathrm{SD}_{\mathrm{b}}}<\gamma_{\mathrm{th}}\right)}_{\Omega} \sum_{m=1}^{M} \underbrace{\operatorname{Pr}\left(\mathrm{D}_{\mathrm{b}}=\mathrm{D}_{m}\right)}_{\Theta_{m}} \\
& \times \prod_{k=1}^{K} \underbrace{\operatorname{Pr}\left(\frac{\alpha\left|h_{\mathrm{SR}_{k}}\right|^{2} \beta\left|h_{\mathrm{SR}_{k}}\right|^{2}\left|h_{\mathrm{R}_{k} \mathrm{D}_{m}}\right|^{2}}{\kappa_{k}\left|h_{\mathrm{SR}_{k}}\right|^{2}+\beta\left|h_{\mathrm{SR}_{k}}\right|^{2}\left|h_{\mathrm{R}_{k} \mathrm{D}_{m}}\right|^{2}}<\gamma_{\mathrm{th}}\right)}_{\mathrm{C}} .
\end{aligned}
$$

As can be seen that $C$ in (74) has a similar form of $B$ in (70). Thus, by following the same calculation manner, we have $\mathrm{C}=$ $1-\lambda_{\mathrm{SR}_{k}} \int_{\mu}^{\infty} e^{-y \lambda_{\mathrm{SR}_{k}}-\frac{\kappa_{k} \gamma_{\mathrm{th}} \mathrm{R}_{k} \mathrm{D}_{m}}{\left(\alpha y-\gamma_{\mathrm{th}}\right) \beta}} d y$. Now, applying Lemma 2 for the case of $a=\lambda_{\mathrm{SR}_{k}}, b=\kappa_{k} \gamma_{\mathrm{th}} \lambda_{\mathrm{R}_{k} \mathrm{D}_{m}}, c=\alpha \beta$, and $d=\gamma_{\mathrm{th}} \beta$, and after some manipulation steps, (26) can be obtained. This completes the proof of Theorem 3 .

\section{APPENDIX D \\ PROOF OF THEOREM 4}

Since the direct links are independent with the dual-hop links, (27) can be re-expressed as

$$
\begin{aligned}
P_{\text {out }}^{\mathrm{DPS}, \mathrm{DF}}= & \underbrace{\operatorname{Pr}\left(\gamma_{\mathrm{SD}_{\mathrm{b}}}<\gamma_{\mathrm{th}}\right)}_{\Omega} \\
& \times \underbrace{\operatorname{Pr}\left(\min \left\{\alpha\left|h_{\mathrm{SR}_{\mathrm{b}}}\right|^{2}, \beta\left|h_{\mathrm{SR}_{\mathrm{b}}}\right|^{2}\left|h_{\mathrm{R}_{\mathrm{b}} \mathrm{D}_{\mathrm{b}}}\right|^{2}\right\}<\gamma_{\mathrm{th}}\right)}_{\mathrm{D}} .
\end{aligned}
$$

For the sake of notational convenience, let $X \triangleq\left|h_{\mathrm{SR}_{\mathrm{b}}}\right|^{2}$, and by conditioning on $X=x, \mathrm{D}$ in (75) can be re-expressed as

$$
\begin{aligned}
\mathrm{D}= & \int_{0}^{\infty}\left(1-\operatorname{Pr}\left(\alpha x \geq \gamma_{\mathrm{th}}\right) \operatorname{Pr}\left(\beta x\left|h_{\mathrm{R}_{\mathrm{b}} \mathrm{D}_{\mathrm{b}}}\right|^{2} \geq \gamma_{\mathrm{th}}\right)\right) \\
& \times f_{X}(x) d x
\end{aligned}
$$

where $f_{X}(x)$ denotes the PDF of $X$. Since $\int_{0}^{\infty} f_{X}(x) d x=1$. By using the total probability theorem [43], D in (76) can be given by

$$
\begin{aligned}
\mathrm{D}= & 1-\sum_{m=1}^{M} \underbrace{\operatorname{Pr}\left(\mathrm{D}_{\mathrm{b}}=\mathrm{D}_{m}\right)}_{\Theta_{m}} \sum_{k=1}^{K} \underbrace{\operatorname{Pr}\left(\mathrm{R}_{\mathrm{b}}=\mathrm{R}_{k}\right)}_{\Xi_{k}} \\
& \times \int_{\mu}^{\infty}\left(1-F_{\left|h_{\mathrm{R}_{k} \mathrm{D}_{m}}\right|^{2}}\left(\frac{\gamma_{\mathrm{th}}}{\beta x}\right)\right) f_{X}(x) d x,
\end{aligned}
$$

where $\mu=\gamma_{\mathrm{th}} / \alpha$. Since $\left|h_{\mathrm{R}_{k} \mathrm{D}_{m}}\right|^{2}$ follows exponential distribution with parameter $\lambda_{\mathrm{R}_{k} \mathrm{D}_{m}}$, and invoking the PDF of $X=\left|h_{\mathrm{SR}_{\mathrm{b}}}\right|^{2}$ in Lemma 3, D can be further expressed as

$$
\mathrm{D}=1-\sum_{m=1}^{M} \Theta_{m} \sum_{k=1}^{K} \Xi_{k} \int_{\mu}^{\infty} e^{-\frac{\gamma_{t h}}{\beta x} \lambda_{\mathrm{R}_{k} \mathrm{D} m}} \widetilde{\sum_{l}} \widehat{\sum_{t}} e^{-x \widehat{\sum}_{t}} d x
$$

Plugging (67) and (68) into (78), and then combining with (66), (31) can be obtained. This completes the proof of Theorem 4.

\section{APPENDIX E \\ PROOF OF THEOREM 5}

Since the direct links are independent with the dual-hop links, and using (8) and (9), (30) can be rewritten as

$$
\begin{aligned}
P_{\text {out }}^{\text {DPS, }} & \text { VG-AF } \\
= & \underbrace{\operatorname{Pr}\left(\gamma_{\mathrm{SD}_{\mathrm{b}}}<\gamma_{\mathrm{th}}\right)}_{\Omega} \\
& \times \operatorname{Pr}\left(\frac{\alpha\left|h_{\mathrm{SR}_{\mathrm{b}}}\right|^{2} \beta\left|h_{\mathrm{SR}_{\mathrm{b}}}\right|^{2}\left|h_{\mathrm{R}_{\mathrm{b}} \mathrm{D}_{\mathrm{b}}}\right|^{2}}{\alpha\left|h_{\mathrm{SR}_{\mathrm{b}}}\right|^{2}+\beta\left|h_{\mathrm{SR}_{\mathrm{b}}}\right|^{2}\left|h_{\mathrm{R}_{\mathrm{b}} \mathrm{D}_{\mathrm{b}}}\right|^{2}+1}<\gamma_{\mathrm{th}}\right) .
\end{aligned}
$$


Let $X \triangleq\left|h_{\mathrm{SR}_{\mathrm{b}}}\right|^{2}$, using the total probability theorem [43], (79) can be further expressed as

$$
\begin{aligned}
P_{\text {out }}^{\text {DPS, VG-AF }} & \\
= & \Omega \sum_{m=1}^{M} \underbrace{\operatorname{Pr}\left(\mathrm{D}_{\mathrm{b}}=\mathrm{D}_{m}\right)}_{\Theta_{m}} \sum_{k=1}^{K} \\
& \times \underbrace{\operatorname{Pr}\left(\mathrm{R}_{\mathrm{b}}=\mathrm{R}_{k}\right)}_{\Xi_{k}} \underbrace{\operatorname{Pr}\left(\frac{\alpha \beta X^{2} \mid h_{\left.\mathrm{R}_{k} \mathrm{D}_{m}\right|^{2}}}{\alpha X+\beta X\left|h_{\mathrm{R}_{k} \mathrm{D}_{m}}\right|^{2}+1}<\gamma_{\mathrm{th}}\right)}_{\mathrm{E}} .
\end{aligned}
$$

Note that in (80), the statistical characteristic of $X$ is still remained, i.e., $X=\left|h_{\mathrm{SR}_{\mathrm{b}}}\right|^{2}=\max _{1 \leq k \leq K}\left\{\left|h_{\mathrm{SR}_{k}}\right|^{2}\right\}$ since the selection criterion of the DPS scheme selects the best relay first, then computes the end-to-end SNR of the selected dualhop link.

As can be seen, $E$ in (80) has a similar form of B in (70). Thus, by following the same calculation manner, and since $\left|h_{\mathrm{R}_{k} \mathrm{D}_{m}}\right|^{2}$ follows an exponential distribution with parameter $\lambda_{\mathrm{R}_{k} \mathrm{D}_{m}}$, and invoking the PDF $f_{X}(x)$ of $X=\left|h_{\mathrm{SR}_{\mathrm{b}}}\right|^{2}$ in Lemma 3, after some algebraic manipulations, (31) can be obtained. This completes the proof of Theorem 5 .

\section{APPENDIX F \\ PROOF OF THEOREM 6}

Since the direct links are independent with the dual-hop links, and from (8) and (9), $P_{\text {out,exact }}^{\mathrm{DPS}, \mathrm{FG}-\mathrm{AF}}$ in (34) can be expressed as

$$
\begin{aligned}
P_{\text {out,exact }}^{\text {DPS }} & \text { AF } \\
= & \underbrace{\operatorname{Pr}\left(\gamma_{\mathrm{SD}_{\mathrm{b}}}<\gamma_{\mathrm{th}}\right)}_{\Omega} \\
& \times \underbrace{\operatorname{Pr}\left(\frac{\alpha\left|h_{\mathrm{SR}_{\mathrm{b}}}\right|^{2} \beta\left|h_{\mathrm{SR}_{\mathrm{b}}}\right|^{2}\left|h_{\mathrm{R}_{\mathrm{b}} \mathrm{D}_{\mathrm{b}}}\right|^{2}}{\kappa_{\mathrm{b}}\left|h_{\mathrm{SR}_{\mathrm{b}}}\right|^{2}+\beta\left|h_{\mathrm{SR}_{\mathrm{b}}}\right|^{2}\left|h_{\mathrm{R}_{\mathrm{b}} \mathrm{D}_{\mathrm{b}}}\right|^{2}}<\gamma_{\mathrm{th}}\right)}_{\mathrm{F}} .
\end{aligned}
$$

By carrying out the similar steps as done in Appendix E, F can be given by

$\mathrm{F}=\sum_{m=1}^{M} \Theta_{m} \sum_{k=1}^{K} \Xi_{k}\left[1-\widetilde{\sum_{l}} \widehat{\sum_{t}} \int_{\mu}^{\infty} e^{-x \widehat{\sum}_{t}-\frac{\kappa_{k} \gamma_{\mathrm{th}} \lambda_{\mathrm{R}_{k} \mathrm{D} m}}{\beta\left(\alpha x-\gamma_{\mathrm{th}}\right)}} d x\right]$.

Applying Lemma 2 to (82) with $a=\widehat{\sum}_{t}, b=\kappa_{k} \gamma_{\mathrm{th}} \lambda_{\mathrm{R}_{k} \mathrm{D}_{m}}$, $c=\alpha \beta$, and $d=\gamma_{\mathrm{th}} \beta$, and then combining with (81), (35) can be obtained. This completes the proof of Theorem 6 .

\section{REFERENCES}

[1] N. T. Do, D. B. da Costa, T. Q. Duong, V. N. Q. Bao, and B. An, "Opportunistic scheduling for fixed-gain amplify-and-forward-based multiuser multirelay SWIPT cooperative networks," in 2017 Int. Conf. on Recent Advances in Signal Process. Telecommunications Computing (SigTelCom), Da Nang, Vietnam, 2017, pp. 49-54.

[2] X. Lu, P. Wang, D. Niyato, D. I. Kim, and Z. Han, "Wireless networks with RF energy harvesting: A contemporary survey," IEEE Commun. Surveys Tuts., vol. 17, no. 2, pp. 757-789, Second quarter 2015.

[3] I. Krikidis, S. Timotheou, S. Nikolaou, G. Zheng, D. W. K. Ng, and R. Schober, "Simultaneous wireless information and power transfer in modern communication systems," IEEE Commun. Mag., vol. 52, no. 11, pp. 104-110, Nov. 2014.
[4] L. R. Varshney, "Transporting information and energy simultaneously," in Proc. IEEE ISIT, Toronto, ON, Canada, Jul. 2008, pp. 1612-1616.

[5] P. Grover and A. Sahai, "Shannon meets tesla: wireless information and power transfer," in Proc. IEEE ISIT, Austin, TX, USA, Jun. 2010, pp. $2363-2367$.

[6] K. Huang and E. Larsson, "Simultaneous information and power transfer for broadband wireless systems," IEEE Trans. Signal Processing, vol. 61 , no. 23, pp. 5972-5986, Dec. 2013.

[7] X. Zhou, R. Zhang, and C. K. Ho, "Wireless information and power transfer: architecture design and rate-energy tradeoff," IEEE Trans. Commun., vol. 61, no. 11, pp. 4754-4767, Nov. 2013.

[8] V. Genc, S. Murphy, Y. Yu, and J. Murphy, "IEEE 802.16j relaybased wireless access networks: an overview," IEEE Wireless Commun. vol. 15 , no. 5, pp. 56-63, Oct. 2008.

[9] T. Q. Duong, D. B. da Costa, M. Elkashlan, and V. N. Q. Bao, "Cognitive amplify-and-forward relay networks over nakagami- $m$ fading," IEEE Trans. Veh. Technol., vol. 61, no. 5, pp. 2368-2374, Jun. 2012.

[10] V. N. Q. Bao, T. Q. Duong, D. B. da Costa, G. C. Alexandropoulos, and A. Nallanathan, "Cognitive amplify-and-forward relaying with best relay selection in non-identical rayleigh fading," IEEE Commun. Lett., vol. 17, no. 3, pp. 475-478, Mar. 2013.

[11] K. Xiong, P. Fan, H. C. Yang, and K. B. Letaief, "Space-time network coding with overhearing relays," IEEE Trans. Wireless Commun., vol. 13, no. 7, pp. 3567-3582, Jul. 2014

[12] Q. Zhou and H. Dai, "Asymptotic analysis on the interaction between spatial diversity and multiuser diversity in wireless networks," IEEE Trans. Signal Processing, vol. 55, no. 8, pp. 4271-4283, Aug. 2007.

[13] N. Yang, M. Elkashlan, and J. Yuan, "Outage probability of multiuser relay networks in nakagami- $m$ fading channels," IEEE Trans. Veh. Technol., vol. 59, no. 5, pp. 2120-2132, Jun. 2010.

[14] H. Ding, J. Ge, D. B. da Costa, and Y. Guo, "Spectrally efficient diversity exploitation schemes for downlink cooperative cellular networks," IEEE Trans. Veh. Technol., vol. 61, no. 1, pp. 386-393, Jan. 2012.

[15] A. A. Nasir, X. Zhou, S. Durrani, and R. A. Kennedy, "Relaying protocols for wireless energy harvesting and information processing," IEEE Trans. Wireless Commun., vol. 12, no. 7, pp. 3622-3636, Jul. 2013.

[16] M. Ju, K. M. Kang, K. S. Hwang, and C. Jeong, "Maximum transmission rate of PSR/TSR protocols in wireless energy harvesting DF-based relay networks," IEEE J. Select. Areas Commun., vol. 33, no. 12, pp. 27012717, Dec. 2015.

[17] K. Xiong, P. Fan, C. Zhang, and K. B. Letaief, "Wireless information and energy transfer for two-hop non-regenerative MIMO-OFDM relay networks," IEEE J. Select. Areas Commun., vol. 33, no. 8, pp. 15951611, Aug. 2015.

[18] D. B. da Costa and S. Aissa, "Capacity analysis of cooperative systems with relay selection in Nakagami- $m$ fading," IEEE Commun. Lett., vol. 13, no. 9, pp. 637-639, Sep. 2009.

[19] T. Q. Duong, V. N. Q. Bao, and H. J. Zepernick, "On the performance of selection decode-and-forward relay networks over Nakagami- $m$ fading channels," IEEE Commun. Lett., vol. 13, no. 3, pp. 172-174, Mar. 2009.

[20] I. H. Lee and D. Kim, "Outage performance of opportunistic cooperation in amplify-and-forward relaying systems with relay selection," IEEE Commun. Lett., vol. 16, no. 2, pp. 224-227, Feb. 2012.

[21] B. Zhang, M. El-Hajjar, and L. Hanzo, "Opportunistic relay selection for cooperative relaying in cochannel interference contaminated networks," IEEE Trans. Veh. Technol., vol. 63, no. 5, pp. 2455-2461, Jun. 2014.

[22] I. Krikidis, "Relay selection in wireless powered cooperative networks with energy storage," IEEE J. Select. Areas Commun., vol. 33, no. 12, pp. 2596-2610, Dec. 2015.

[23] K. H. Liu, "Performance analysis of relay selection for cooperative relays based on wireless power transfer with finite energy storage," IEEE Trans. Veh. Technol., vol. 65, no. 7, pp. 5110-5121, Jul. 2016.

[24] Z. Ding, S. M. Perlaza, I. Esnaola, and H. V. Poor, "Power allocation strategies in energy harvesting wireless cooperative networks," IEEE Trans. Wireless Commun., vol. 13, no. 2, pp. 846-860, Feb. 2014.

[25] Z. Ding and H. V. Poor, "Multi-user SWIPT cooperative networks: Is the max-min criterion still diversity-optimal?" IEEE Trans. Wireless Commun., vol. 15, no. 1, pp. 553-567, Jan. 2016.

[26] H. Chen, Y. Li, Y. Jiang, Y. Ma, and B. Vucetic, "Distributed power splitting for SWIPT in relay interference channels using game theory," IEEE Trans. Wireless Commun., vol. 14, no. 1, pp. 410-420, Jan. 2015.

[27] A. A. Nasir, D. T. Ngo, X. Zhou, R. A. Kennedy, and S. Durrani, "Joint resource optimization for multicell networks with wireless energy harvesting relays," IEEE Trans. Veh. Technol., vol. 65, no. 8, pp. 61686183, Aug. 2016. 
[28] H. Chen, X. Zhou, Y. Li, P. Wang, and B. Vucetic, "Wireless-powered cooperative communications via a hybrid relay," in 2014 IEEE Inform. Theory Workshop (ITW), Nov. 2014, pp. 666-670.

[29] H. Chen, Y. Li, J. L. Rebelatto, B. F. Ucha-Filho, and B. Vucetic, "Harvest-then-cooperate: Wireless-powered cooperative communications," IEEE Trans. Signal Processing, vol. 63, no. 7, pp. 1700-1711, Apr. 2015.

[30] Z. Ding, I. Krikidis, B. Sharif, and H. V. Poor, "Wireless information and power transfer in cooperative networks with spatially random relays," IEEE Trans. Wireless Commun., vol. 13, no. 8, pp. 4440-4453, Aug. 2014.

[31] Y. Gu and S. Aissa, "RF-based energy harvesting in decode-and-forward relaying systems: ergodic and outage capacities," IEEE Trans. Wireless Commun., vol. 14, no. 11, pp. 6425-6434, Nov. 2015.

[32] N. I. Miridakis, T. A. Tsiftsis, G. C. Alexandropoulos, and M. Debbah, "Energy efficient switching between data transmission and energy harvesting for cooperative cognitive relaying systems," in 2016 IEEE Int. Conf. Commun. (ICC), May 2016, pp. 1-6.

[33] I. Krikidis, "Simultaneous information and energy transfer in large-scale networks with/without relaying," IEEE Trans. Commun., vol. 62, no. 3 , pp. 900-912, Mar. 2014.

[34] R. Zhang and C. K. Ho, "MIMO broadcasting for simultaneous wireless information and power transfer," IEEE Trans. Wireless Commun., vol. 12, no. 5, pp. 1989-2001, May 2013.

[35] G. Zhu, C. Zhong, H. A. Suraweera, G. K. Karagiannidis, Z. Zhang, and T. A. Tsiftsis, "Wireless information and power transfer in relay systems with multiple antennas and interference," IEEE Trans. Commun., vol. 63, no. 4, pp. 1400-1418, Apr. 2015.

[36] L. Liu, R. Zhang, and K. C. Chua, "Wireless information and power transfer: a dynamic power splitting approach," IEEE Trans. Commun., vol. 61, no. 9, pp. 3990-4001, Sep. 2013.

[37] M. O. Hasna and M. S. Alouini, "A performance study of dual-hop transmissions with fixed gain relays," IEEE Trans. Wireless Commun., vol. 3, no. 6, pp. 1963-1968, Nov. 2004.

[38] Y. Gu, H. Chen, Y. Li, and B. Vucetic, "Distributed multi-relay selection in accumulate-then-forward energy harvesting relay networks," 2016. [Online]. Available: https://arxiv.org/abs/1602.00339

[39] I. S. Gradshteyn and I. M. Ryzhik, Tables of Integrals, Series, and Products 7th Ed. New York, NY, USA: Academic Press, 2007.

[40] S. Boyd and L. Vandenberghe, Convex Optimization. Cambridge, U.K.: Cambridge Univ. Press, 2004

[41] L. Zheng and D. N. C. Tse, "Diversity and multiplexing: a fundamental tradeoff in multiple-antenna channels," IEEE Trans. Inf. Theory, vol. 49, no. 5, pp. 1073-1096, May 2003.

[42] Y. Liu, Z. Ding, M. Elkashlan, and H. V. Poor, "Cooperative nonorthogonal multiple access with simultaneous wireless information and power transfer," IEEE J. Select. Areas Commun., vol. 34, no. 4, pp. 938-953, Apr. 2016.

[43] A. Papoulis and S. U. Pillai, Probability, random variables, and stochastic processes, 4th ed. New York, NY, USA: McGraw-Hill, 2002, pp. 101-105.

[44] H. Ding, J. Ge, D. B. da Costa, and Z. Jiang, "A new efficient lowcomplexity scheme for multi-source multi-relay cooperative networks," IEEE Trans. Veh. Technol., vol. 60, no. 2, pp. 716-722, Feb. 2011.

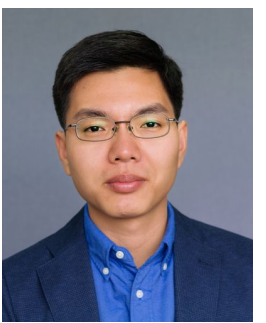

Nhu Tri Do (S'16) was born in Da Nang, Vietnam. He attended Le Quy Don high school, Da Nang in 2007. He received the B.S. degree in electronics and telecommunications engineering from the Posts and Telecommunications Institute of Technology (PTIT), Saigon, Vietnam in 2012. He received the M.S. degree in electronics and computer engineering from Hongik University, Sejong campus, South Korea in 2015. He is currently working as Ph.D. student and Teaching Associate in electronics and computer engineering at Hongik University, Sejong campus, South Korea. His main research topics are: wireless communications and cooperative relaying transmissions.

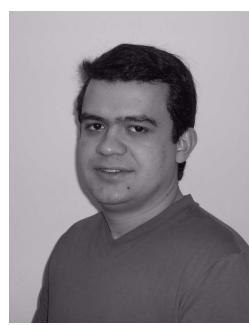

Daniel Benevides da Costa (S'04 - M'08 - SM'14) was born in Fortaleza, Ceará, Brazil, in 1981. He received the B.Sc. degree in Telecommunications from the Military Institute of Engineering (IME), Rio de Janeiro, Brazil, in 2003, and the M.Sc. and $\mathrm{Ph} . \mathrm{D}$. degrees in Electrical Engineering, Area: Telecommunications, from the University of Campinas, SP, Brazil, in 2006 and 2008, respectively. His Ph.D thesis was awarded the Best Ph.D. Thesis in Electrical Engineering by the Brazilian Ministry of Education (CAPES) at the 2009 CAPES Thesis Contest. From 2008 to 2009, he was a Postdoctoral Research Fellow with INRS-EMT, University of Quebec, Montreal, QC, Canada. Since 2010, he has been with the Federal University of Ceará, where he is currently an Assistant Professor. Prof. da Costa is currently Editor of the IEEE COMMUNICATIONS SURVEYS AND TUTORIALS, IEEE ACCESS, IEEE COMMUNICATIONS LETTERs, IEEE TRANSACTIONS ON Vehicular TEChNOLOGY, EURASIP JOURNAL ON WIRELESS COMMUNICATIONS AND NETWORKING, and KSII TRANSACTIONS ON INTERNET AND INFORMATION SYSTEMS. He has also served as Associate Technical Editor of the IEEE COMMUNICATIONS MAGAZINE. He has served as the Lead Guest Editor and Guest Editor of several Journal Special Issues. He has been involved on the organization of several conferences. In particular, he served as Track Chair (Track 6 - Green Communications and Networks; Track - Recent Results on 5G) of the IEEE 85th Vehicular Technology Conference (VTC2017-Spring), and is currently serving as Track Co-Chair (Track 1 - Antenna Systems, Propagation, and RF Design) of the IEEE VTC2017-Fall. He is currently the Latin American Chapters Coordinator of the IEEE Vehicular Technology Society. Also, he acts as a Scientific Consultant of the National Council of Scientific and Technological Development (CNPq), Brazil and he is a Productivity Research Fellow of CNPq. From 2012 to 2017, he was Member of the Advisory Board of the Cear Council of Scientific and Technological Development (FUNCAP), Area: Telecommunications. Prof. da Costa is the recipient of three conference paper awards. He received the Exemplary Reviewer Certificate of the IEEE WiRELESS COMMUNICATIONS LETTERS in 2013, the Exemplary Reviewer Certificate of the IEEE COMMUNiCATIONS LETTERS in 2016, the Certificate of Appreciation of Top Associate Editor for outstanding contributions to IEEE TRANSACTIONS ON VEHICULAR TECHNOLOGY in 2013, 2015 and 2016, and the Exemplary Editor Award of IEEE COMMUNICATIONS LETTERS in 2016. $\mathrm{He}$ is a Senior Member of IEEE, Member of IEEE Communications Society and IEEE Vehicular Technology Society.

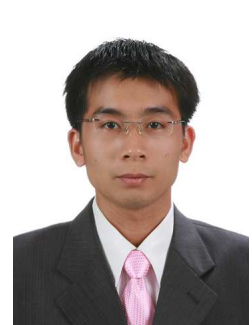

Trung Q. Duong (S'05, M'12, SM'13) received his $\mathrm{Ph} . \mathrm{D}$. degree in Telecommunications Systems from Blekinge Institute of Technology (BTH), Sweden in 2012. Since 2013, he has joined Queen's University Belfast, UK as a Lecturer (Assistant Professor). His current research interests include small-cell networks, physical layer security, energy-harvesting communications, cognitive relay networks. $\mathrm{He}$ is the author or co-author of more than 250 technical papers published in scientific journals (138 articles) and presented at international conferences $(120 \mathrm{pa}-$

pers).

Dr. Duong currently serves as an Editor for the IEEE TRANSACTIONS on Wireless COMMUNiCATIONS, IEEE TRANSACTIONS ON COMMUNICATIONS, IET COMMUNICATIONS, and a Senior Editor for IEEE COMMUNiCATIONS LETTERs. He was awarded the Best Paper Award at the IEEE Vehicular Technology Conference (VTC-Spring) in 2013, IEEE International Conference on Communications (ICC) 2014, and IEEE Global Communications Conference (GLOBECOM) 2016. He is the recipient of prestigious Royal Academy of Engineering Research Fellowship (2016-2021). 


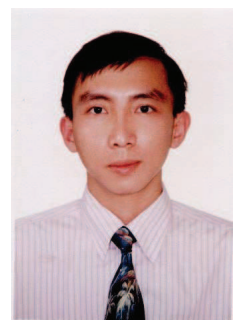

Vo Nguyen Quoc Bao (M'11, SM'16) is an associate professor of Wireless Communications at Posts and Telecommunications Institute of Technology (PTIT), Vietnam. He also served as the Dean of Faculty of Telecommunications and a Director of the Wireless Communication Laboratory (WCOMM). His research interests include wireless communications and information theory with current emphasis on MIMO systems, cooperative and cognitive communications, physical layer security, and energy harvesting. He has published more than 140 journal and conference articles that have $1500+$ citations and $\mathrm{H}$-index of $21 . \mathrm{He}$ is an Associate Editor of EURASIP Journal on Wireless Communications and Networking, an Editor of Transactions on Emerging Telecommunications Technologies (Wiley ETT), VNU Journal of Computer Science and Communication Engineering, and REV Journal on Electronics and Communications. $\mathrm{He}$ served as a Technical Program co-chair for ATC (2013, 2014), NAFOSTEDNICS (2014, 2015, 2016), REV-ECIT 2015, ComManTel (2014, 2015), and SigComTel 2017. He is a Member of the Executive Board of the RadioElectronics Association of Vietnam (REV) and the Electronics Information and Communications Association Ho Chi Minh City (EIC). He is currently serving as a scientific secretary of the Vietnam National Foundation for Science and Technology Development (NAFOSTED) scientific Committee in Information Technology and Computer Science.

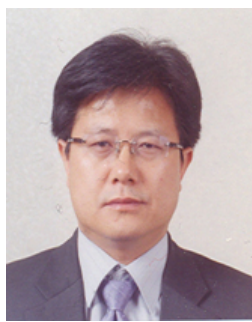

Beongku An received the M.S. degree in electrical engineering from the New York University (Polytechnic), NY, USA, in 1996 and Ph.D. degree from New Jersey Institute of Technology (NJIT), NJ, USA, in 2002, BS degree in electronic engineering from Kyungpook National university, Korea, in 1988, respectively. After graduation, he joined the Faculty of the Department of Computer and Information Communications Engineering, Hongik University in Korea, where he is currently a Professor. From 1989 to 1993, he was a senior researcher in RIST, Pohang, Korea. He also was lecturer and RA in NJIT from 1997 to 2002. He was a president of IEIE Computer Society (The Institute of Electronics and Information Engineers, Computer Society) in 2012. From 2013, he also works as a General Chair in the International Conference, ICGHIT (International Conference on Green and Human Information Technology). His current research interests include mobile wireless networks and communications such as ad-hoc networks, sensor networks, wireless internet, cognitive radio networks, ubiquitous networks, cellular networks, and IoT. In particular, he is interested in cooperative routing, multicast routing, energy harvesting, physical layer security, visible light communication (VLC), cross-layer technology, mobile cloud computing. Professor An was listed in Marquis Whos Who in Science and Engineering, and Marquis Whos Who in the World, respectively. 